Y. Miyashita

Nagoya Math. J.

Vol. 49 (1973), 21-51

\title{
AN EXACT SEQUENCE ASSOCIATED WITH A GENERALIZED CROSSED PRODUCT
}

\author{
YÔICHI MIYASHITA
}

\section{§ 0. Introduction}

The purpose of this paper is to generalize the seven terms exact sequence given by Chase, Harrison and Rosenberg [8]. Our work was motivated by Kanzaki [16] and, of course, [8], [9]. The main theorem holds for any generalized crossed product, which is a more general one than that in Kanzaki [16]. In $\S 1$, we define a group $P(A / B)$ for any ring extension $A / B$, and prove some preliminary exact sequences. In $\S 2$, we fix a group homomorphism $J$ from a group $G$ to the group of all invertible two-sided $B$-submodules of $A$. We put $\Delta / B=\oplus J_{\sigma} / B$ (direct sum), which is canonically a generalized crossed product of $B$ with $G$. And we define an abelian group $C(\Delta / B)$ for $\Delta / B$. The two groups $C(\Delta / B)$ and $P(A / B)$ are our main objects. $C(\Delta / B)$ may be considered as a generalization of the group of all central separable algebras split by a fixed Galois extension. The main theorem is Th. 2.12, which is a generalization of the seven terms exact sequence theorem in [8]. However it is proved that the exact sequence in Th. 2.12 is almost reduced to the one which is obtained from the homomorphism $G \rightarrow \operatorname{Aut}(K)$ induced by $J$, where $K$ is the center of $B$. This fact is proved in Th. 2.15. In $\S 3$, we fix a group homomorphism $u: G \rightarrow \operatorname{Aut}(A / B)$. From $u$ we obtain a free crossed product $\oplus A u_{\sigma} / B$, where $u_{\sigma} u_{\tau}=u_{\sigma}, u_{\sigma} a=\sigma(a) u_{\sigma}(a \in A)$. Therefore the results in $\S 2$ is applicable for this case. In $\S 4$ we prove the Morita invariance of the exact sequence in Th. 2.12. In $\S 5$, we treat a kind of duality, which is based on a result obtained in [19]. In $\S 6$ we study the splitting of $P(A / B)$ in particular cases.

Received December 23, 1971. 


\section{$\S 1$. The definition of $P(A / B)$, and related exact sequences.}

As to notations and terminologies used in this paper we follow [19], unless otherwise expressed.

Let $G, G^{\prime}$ be groups, and $f$ a homomorphism from $G$ to the group of all automorphisms of $G^{\prime}$. Then $G$ operates on $G^{\prime}$, by $f$. Then we call $G^{\prime}$ a $G$-group. We denote by $G^{\prime G}$ the subgroup $\left\{g^{\prime} \in G^{\prime} \mid g\left(g^{\prime}\right)=g^{\prime}\right.$ for all $g \in G\}$.

Let $A \supseteq B$ be rings with common identity, and let $L, K$ be the centers of $A$ and $B$, respectively. We denote by $\circlearrowleft(A / B)$ the group of all invertible two-sided $B$-submodules of $A$ (cf. [19]), where a two-sided $B$-submodule $X$ of $A$ is invertible in $A$ if and only if $X Y=Y X=B$ for some $B$ - $B$-submodule $Y$ of $A$. We denote by Aut $(A / B)$ the group of all $B$-automorphisms of a ring $A$, which operates on the left. Then it is evident that $\&(A / B)$ is canonically a left Aut $(A / B)$-group. On the other hand we have

Proposition 1.1. Aut $(A / B)$ is a $\leftrightarrow(A / B)$-group.

Proof. Let $X$ be in $\varangle(A / B)$. Then $A=X A=X \otimes{ }_{B} A=A X^{-1}=$ $A \otimes{ }_{B} X^{-1}$ canonically (cf. [19; Prop. 1.1]), and hence $X \otimes{ }_{B} A \otimes{ }_{B} X^{-1} \rightarrow$ $A, x \otimes a \otimes x^{\prime} \mapsto x a x^{\prime}$ is an isomorphism. Therefore, for any $\sigma$ in $\operatorname{Aut}(A / B)$, the mapping $X(\sigma): x \otimes a \otimes x^{\prime} \mapsto x \otimes \sigma(a) \otimes x^{\prime}\left(x \in X, x^{\prime} \in X^{-1}\right)$ from $A$ to $A$ is well defined. Then it is easily seen that $X(\sigma)$ is a $B$-automorphism of $A$, and this defines a $\&(A / B)$-group Aut $(A / B)$.

Here we continue the study of $X(\sigma)$ for the sequel. Since $X X^{-1}=$ $B \ni 1,1$ is written as $1=\sum_{i} a_{i} a_{i}^{\prime}\left(a_{i} \in X, a_{i}^{\prime} \in X^{-1}\right)$. Then $\sum_{i} \tau\left(a_{i}\right) \sigma\left(a_{i}^{\prime}\right)$ $\cdot \sum_{i} \sigma\left(a_{i}\right) \tau\left(a_{i}^{\prime}\right)=1$ for $\sigma, \tau$ in Aut $(A / B)$. Since $\sum_{i} a_{i} \otimes a_{i}^{\prime} \mapsto 1$ under the isomorphism $X \otimes{ }_{B} X^{-1} \rightarrow B$, we know that $\sum_{i} b a_{i} \otimes a_{i}^{\prime}=\sum_{i} a_{i} \otimes a_{i}^{\prime} b$ for all $b$ in $B$, and so $b \sum_{i} \tau\left(a_{i}\right) \sigma\left(a_{i}^{\prime}\right)=\sum_{i} \tau\left(a_{i}\right) \sigma\left(a_{i}^{\prime}\right) b$. Thus $\sum_{i} \tau\left(a_{i}\right) \sigma\left(a_{i}^{\prime}\right) \epsilon$ $U\left(V_{A}(B)\right)$ (the group of all invertible elements of $\left.V_{A}(B)\right)$, and $\left(\sum_{i} \tau\left(a_{i}\right) \sigma\left(a_{i}^{\prime}\right)\right)^{-1}$ $=\sum_{i} \sigma\left(a_{i}\right) \tau\left(a_{i}^{\prime}\right)$. Put $u=\sum_{i} a_{i} \cdot \sigma\left(a_{i}^{\prime}\right)$. Then, for any a in $A, u \cdot \sigma(a) u^{-1}=$ $\sum_{i, j} a_{i} \cdot \sigma\left(a_{i}^{\prime}\right) \sigma(\alpha) \sigma\left(a_{j}\right) a_{j}^{\prime}=\sum_{i, j} a_{i} \cdot \sigma\left(a_{i}^{\prime} a a_{j}\right) a_{j}^{\prime}=X(\sigma)\left(\sum_{i, j} a_{i} a_{i}^{\prime} a a_{j} a_{j}^{\prime}\right)=X(\sigma)(\alpha)$. Hence $X(\sigma)$ differs from $\sigma$ by the inner automorphism induced by $u$. Therefore $X(\sigma)=\sigma$ is equivalent to that $u$ is in the center $L$ of $A$. To be easily seen, $u \cdot \sigma(x)=x$ for all $x$ in $X$, (and similarly $\sigma\left(x^{\prime}\right) u^{-1}=x^{\prime}$ for all $x^{\prime}$ in $\left.X^{-1}\right)$. Conversely, since the left annihilator of $X$ in $A$ is zero, this characterizes $u$, and hence $u$ is independent of the choice of 
$a_{i}, a_{i}^{\prime}$, and is denoted by $u(X, 1, \sigma)$, in the sequel. As $\sum_{i} \tau\left(a_{i}\right) \sigma\left(a_{i}^{\prime}\right)=$ $\tau\left(\sum_{i} a_{i} \cdot \tau^{-1} \sigma\left(a_{i}^{\prime}\right)\right), \sum_{i} \tau\left(a_{i}\right) \sigma\left(a_{i}^{\prime}\right)$ is also independent of the choice of $a_{i}, a_{i}^{\prime}$, and is denoted by $u(X, \tau, \sigma)$.

LEMMA 1.2. Let ${ }_{B} P_{B^{\prime}}$ and ${ }_{B} P_{B^{\prime}}^{\prime}$ be Morita modules, $A$ and $A^{\prime}$ are over rings of $B$ and $B^{\prime}$, respectively. Let $f_{0}$ be a left $B$, right $B^{\prime}$ isomorphism $P \rightarrow P^{\prime}$, and $f: A \otimes{ }_{B} P \stackrel{\approx}{\longrightarrow} P^{\prime} \otimes{ }_{B^{\prime}} A^{\prime}$ is a $B$ - $B^{\prime}$-isomorphism such that $f(1 \otimes p)=f_{0}(p) \otimes 1$ for all $p \in P$. Assume that $x f^{-1}\left(f(a \otimes p) x^{\prime}\right)$ $=f^{-1}\left(f(x a \otimes p) x^{\prime}\right)$ for all $x, a \in A, x^{\prime} \in A^{\prime}$. Then, if we define $(a \otimes p) * x^{\prime}$ $=f^{-1}\left(f(a \otimes p) x^{\prime}\right)$, then ${ }_{A} A \otimes{ }_{B} P_{A^{\prime}}$ is a Morita module. (cf. [19])

Proof. Put End $\left({ }_{A} A \otimes{ }_{B} P\right) / B^{\prime}=A^{\prime \prime} / B^{\prime}$. Then, by [19; Lemma 3.1], $P \otimes{ }_{B^{\prime}} A^{\prime \prime} \rightarrow A \otimes{ }_{B} P, p \otimes a^{\prime \prime} \mapsto(1 \otimes p) a^{\prime \prime}$ is an isomorphism. On the other hand $f^{-1}: P^{\prime} \otimes{ }_{B^{\prime}} A^{\prime} \rightarrow A \otimes{ }_{B} P, f_{0}(p) \otimes a^{\prime} \mapsto(1 \otimes p) * a^{\prime}(p \in P)$. By hypothesis, the image of $A^{\prime}$ in the endomorphism ring is contained in $A^{\prime \prime}$. And, since $P_{B^{\prime}}$ is a generator, the above two isomorphisms imply that the image of $A^{\prime}$ is equal to $A^{\prime \prime}$.

Next we define a group $P(A / B) . \quad P(A / B)$ consists of all isomorphic classes of left $B$, right $B$-homomorphism $\varphi$ from a Morita module ${ }_{B} P_{B}$ to a Morita module ${ }_{A} N_{A}$ such that the homomorphism $A \otimes{ }_{B} P \rightarrow N$, $a \otimes p \mapsto a \cdot \varphi(p)$ is an isomorphism (cf. [19; §3]). An isomorphism from $\varphi: P \rightarrow N$ to $\varphi^{\prime}: P^{\prime} \rightarrow N^{\prime}$ is a pair $(f, g)$ of isomorphisms such that the diagram

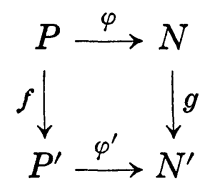

is commutative, where $f$ is a left $B$, right $B$-isomorphism, and $g$ is a left $A$, right $A$-isomorphism. The isomorphism class of $\varphi$ is denoted by [ $\varphi$ ]. The product of $\varphi: P \rightarrow N$ and $\psi: Q \rightarrow U$ is $\varphi \otimes \psi: P \otimes{ }_{B} Q \rightarrow N \otimes{ }_{A} U$, where $(\varphi \otimes \psi)(p \otimes q)=\varphi(p) \otimes \psi(q)$. We define $[\varphi][\psi]=[\varphi \otimes \psi]$. Then this is well-defined, and associative. The inclusion map $B \rightarrow A$ is evidently the identity element. Let $P^{*}=\operatorname{Hom}_{r}\left({ }_{B} P,{ }_{B} B\right.$ ) (cf. [19]), $N^{*}=$ $\operatorname{Hom}_{r}\left({ }_{A} N,{ }_{A} A\right)$, and $\varphi^{*}: P^{*} \rightarrow N^{*}$ the homomorphism such that $\varphi^{*}\left(p^{*}\right)=$ $\left(a \cdot \varphi(p) \rightarrow a \cdot p^{p^{*}}\right)\left(p^{*} \in P^{*}, a \in A, p \in P\right)$ (cf. [19; Lemma 3.1]). Then it is obvious that $\left[\varphi^{*}\right]$ is the inverse element of $[\varphi]$ in $P(A / B)$. Thus we have proved 
THEOREM 1.3. $P(A / B)$ is a group.

Remark. Similarly $P(A / B)$ can be defined for any ring homomorphism $B \rightarrow A$.

THEOREM 1.4. There is an exact sequence

$$
1 \rightarrow U(L) \cap U(K) \rightarrow U(L) \rightarrow \circlearrowleft(A / B) \rightarrow P(A / B) \rightarrow \operatorname{Pic}(A),
$$

where $U\left(^{*}\right)$ is the group of invertible elements of a ring *, and $\operatorname{Pic}(A)$ is the group of isomorphic classes of two-sided A-Morita modules.

Proof. The mapping $U(L) \cap U(K) \rightarrow U(L)$ is the canonical one, and the mapping $U(L) \rightarrow \circlearrowleft(A / B)$ is $c \mapsto B c$. Then $1 \rightarrow U(L) \cap U(K) \rightarrow U(L)$ $\rightarrow \circlearrowleft \&(A / B)$ is evidently exact. For $X$ in $\circlearrowleft(A / B)$, we correspond the canonical inclusion map $i_{X}: X \rightarrow A$. If $i_{X}$ is isomorphic to $i_{B}$, then there is a commutative diagram

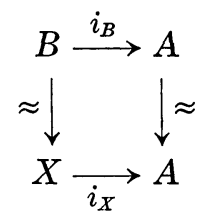

and hence there is an element $d$ in $U(L)$ such that $B d=X$. Hence $U(L) \rightarrow \circlearrowleft(A / B) \rightarrow P(A / B)$ is exact. For $\varphi: P \rightarrow M$ in $P(A / B)$, we correspond $[M]$ (the isomorphic class of $M$ ). If $M \stackrel{\approx}{\longrightarrow} A$ as $A$ - $A$-modules, then we may assume that $M=A$ and $P$ is a $B$ - $B$-submodule of $A$ (cf. [19; Lemma 3.1 (4)]). Then, by [19; Prop. 1.1], we have $P \in \circlearrowleft(A / B)$. This completes the proof.

On the other hand we have

THEOREM 1.5. There is an exact sequence

$$
1 \rightarrow U(L) \cap U(K) \rightarrow U(K) \rightarrow \operatorname{Aut}(A / B) \rightarrow P(A / B) \rightarrow \operatorname{Pic}(B) .
$$

Proof. The map $U(L) \cap U(K) \rightarrow U(K)$ is the canonical one, and the map $U(K) \rightarrow \operatorname{Aut}(A / B)$ is $d \mapsto \tilde{d}$, where $\tilde{d}(a)=d a d^{-1}$ for all $a \in A$. Then $1 \rightarrow U(K) \cap U(L) \rightarrow U(K) \rightarrow \operatorname{Aut}(A / B)$ is evidently exact. For any $\sigma$ in Aut $(A / B)$, we correspond the map $i_{\sigma}: B \rightarrow A u_{\sigma}, b \mapsto b u_{\sigma}$ (cf. [19]). For $d$ in $U(K), d \mapsto \tilde{d} \mapsto i_{\tilde{d}}$. Put $\tilde{d}=\tau$. Then $A \stackrel{\approx}{\longrightarrow} A u_{\tau}, a \mapsto a d^{-1} u_{\tau}$, as $A-A-$ modules, and $B \stackrel{\approx}{\longrightarrow} B$, as $B$-B-modules, by $b \mapsto b d^{-1}$, and we have a commutative diagram 


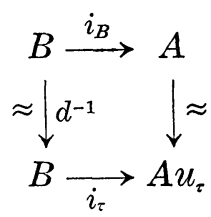

Let $\sigma$ be in Aut $(A / B)$, and suppose that $i_{\sigma}$ is isomorphic to $i_{B}: B \rightarrow A$. Then there are isomorphisms $\alpha, \beta$ such that

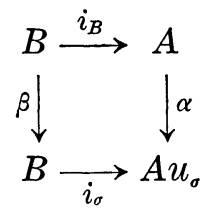

is commutative. Put $\alpha^{-1}\left(u_{\sigma}\right)=d$. Then, for any $a \in A, \sigma(a) d=\alpha^{-1}\left(\sigma(a) u_{\sigma}\right)$ $=\alpha^{-1}\left(u_{\sigma} a\right)=d a$, and so $\sigma(\alpha) d=d a$. Since $\beta(d) u_{\sigma}=\alpha(d)=u_{\sigma}$, we have $\beta(d)=1$, whence $d$ is in $U(K)$, because $\beta$ is a $B$ - $B$-isomorphism. Finally, for $\varphi: P \rightarrow M$ in $P(A / B)$, we correspond $[P] \in \mathrm{Pic}(B)$. If ${ }_{B} B_{B} \stackrel{\approx}{\longrightarrow} P_{B}, 1 \mapsto u$, then $P=B u$ and $M=A \cdot \varphi(u)$. Since $M \stackrel{\approx}{\longrightarrow} A \otimes{ }_{B} P$ as left $A$, right $B$-modules, $a \cdot \varphi(u)=0(a \in A)$ implies $a=0$. Hence there is an automorphism $\sigma \in \operatorname{Aut}(A / B)$ such that $\varphi(u) a=\sigma(a) \varphi(u)$ for all $a \in A$. Then $\varphi$ is isomorphic to $i_{\sigma}$. This completes the proof.

If we cut out $P(A / B)$, we have well known exact sequences.

Proposition 1.6. There are two exact sequences

$$
\begin{aligned}
& 1 \longrightarrow U(K) \longrightarrow U\left(V_{A}(B)\right) \stackrel{\alpha}{\longrightarrow} \circlearrowleft(A / B) \longrightarrow \operatorname{Pic}(B), \\
& 1 \longrightarrow U(L) \longrightarrow U\left(V_{A}(B)\right) \stackrel{\beta}{\longrightarrow} \operatorname{Aut}(A / B) \longrightarrow \operatorname{Pic}(A),
\end{aligned}
$$

where $\alpha(d)=B d$ and $\beta(d)(a)=d a d^{-1}\left(d \in U\left(V_{A}(B)\right), a \in A\right)$.

Here we indicate Th. 1.4, Th. 1.5, and Prop. 1.6 by the following diagram :

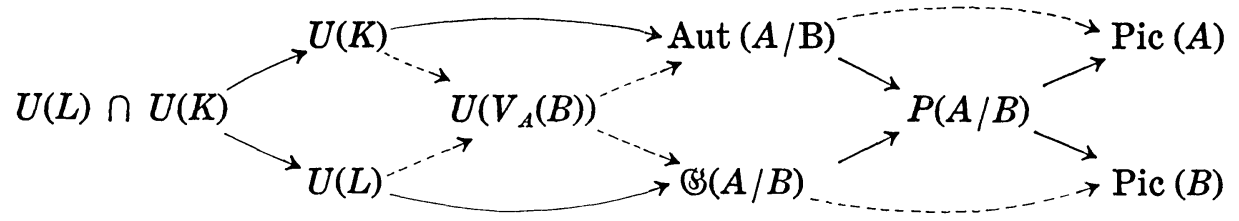

If $A$ is an $R$-algebra, we define $\operatorname{Pic}_{R}(A)=\{[P] \in \operatorname{Pic}(A) \mid r p=p r$ for all $r \in R$ and all $p \in P\}$ and $P^{R}(A / B)=\{[\varphi] \in P(A / B) \mid \varphi: P \rightarrow N,[N] \in$ 
$\left.\operatorname{Pic}_{R}(A)\right\}$. If $B$ is an $S$-algebra, we define $P_{S}(A / B)=\{[\varphi] \in P(A / B) \mid \varphi: P$ $\left.\rightarrow N,[P] \in \operatorname{Pic}_{S}(B)\right\}$.

§ 2. The definition of $C(\Delta / B)$, and an exact sequence associated with $\Delta / B$.

In this section, we fix a (finite or infinite) group $G$, rings $B \subseteq A$, and a group homomorphism $J: \sigma \mapsto J_{\sigma}$ from $G$ to $\mathbb{S}(A / B)$. Then $J$ induces a group homomorphism $G \rightarrow \operatorname{Aut}\left(V_{A}(B) / L\right.$ ) (cf. [19; Prop. 3.3]), and further $G \rightarrow \operatorname{Aut}(K / K \cap L)$. A generalized crossed product $\oplus_{\sigma \in G} J_{\sigma} / B$ associated with $J$ is defined by $\left(x_{\sigma}\right)\left(y_{\sigma}\right)=\left(z_{\sigma}\right)$, where $z_{\sigma}=\sum_{\tau \rho=\sigma} x_{\tau} y_{\rho}$. We denote this by $\Delta / B$ in the sequel. Pic $(B)$ is a left $G$-group defined by ${ }^{\sigma}[P]=\left[J_{\sigma} \otimes{ }_{B} P \otimes{ }_{B} J_{\sigma-1}\right]$ (conjugation). Then we define $\operatorname{Pic}(B)^{G}=\{[P] \in$ $\left.\operatorname{Pic}(B)\right|^{\sigma}[P]=[P]$ for all $\left.\sigma \in G\right\}$, and $\operatorname{Pic}_{K}(B)^{G}=\operatorname{Pic}(B)^{G} \cap \operatorname{Pic}_{K}(B)$. The homomorphism $\mathbb{S}(A / B) \rightarrow P(A / B)$ in Th. 1.4 induces a left $G$-group $P(A / B)$ defined by conjugation.

Proposition 2.1. The following exact sequences consist of G-homomorphisms:

$$
\begin{aligned}
& 1 \longrightarrow U(L) \cap U(K) \longrightarrow U(K) \longrightarrow \operatorname{Aut}(A / B) \longrightarrow P(A / B) \longrightarrow \operatorname{Pic}(B) \\
& 1 \longrightarrow U(L) \longrightarrow U\left(V_{A}(B)\right) \longrightarrow \operatorname{Aut}(A / B) \longrightarrow \operatorname{Pic}(A)
\end{aligned}
$$

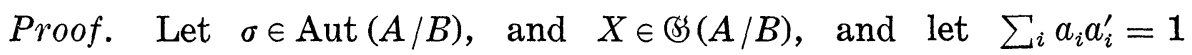
$\left(a_{i} \in X, a_{i}^{\prime} \in X^{-1}\right)$. Then $X(\sigma)(a)=\sum_{i} a_{i} \cdot \sigma\left(a_{i}^{\prime}\right) \sigma(a) \sum_{j} \sigma\left(a_{j}\right) a_{j}^{\prime}$ for all a in $A$ (cf. $\S 1$ ), and so $A u_{\sigma} \stackrel{\approx}{\longrightarrow} A u_{X(\sigma)}$ as $A$-A-modules, by the map $a u_{\sigma} \rightarrow a$ $\cdot \sum_{i} \sigma\left(a_{i}\right) a_{i}^{\prime} u_{X(\sigma)}$. Then the following diagram is commutative:

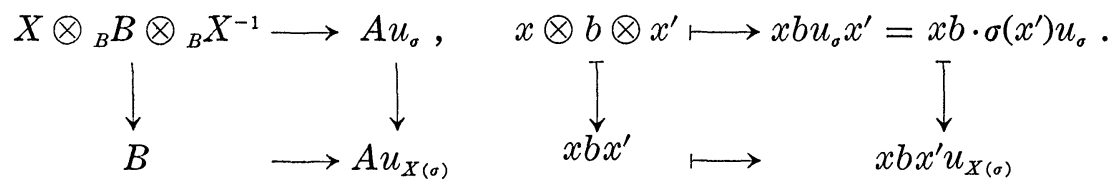

Hence Aut $(A / B) \rightarrow P(A / B)$ is a $G$-homomorphism. Let $c$ be in $U\left(V_{A}(B)\right)$. Then, since $X$ induces an automorphism of $V_{A}(B)$, there is a $c^{\prime} \in U\left(V_{A}(B)\right)$ such that $x c=c^{\prime} x$ for all $x \in X$ (i.e., $X(c)=c^{\prime}$ ). Put $u=\sum_{i} a_{i} \cdot \tilde{c}\left(a_{i}^{\prime}\right)$. Then $c^{\prime} c^{-1} \cdot \tilde{c}(x)=c^{\prime} c^{-1} \cdot c x c^{-1}=c^{\prime} x c^{-1}=x$ for all $x$ in $X$. Hence we know that $c^{\prime} c^{-1}=u$ (cf. $\S 1$ ). For any a in $A, X(\tilde{c})(a)=u \cdot \tilde{c}(a) u^{-1}=c^{\prime} c^{-1} c a c^{-1}$ $\cdot c c^{-1}=c^{\prime} a c^{\prime-1}$. Hence $X(\tilde{c})=\widetilde{c}^{\prime}=\widetilde{X(c)}$. The remainder is obvious.

We define $P(A / B)^{(G)}=\left\{[\phi] \in P(A / B) \mid \phi: P \rightarrow M, J_{\sigma} \cdot \phi(P)=\phi(P) \cdot J_{\sigma}\right.$ for all $\sigma \in G\}$. Then $P(A / B)^{(G)}$ is a subgroup of $P(A / B)^{G}$. In fact, for $\phi: P \rightarrow M$ in $P(A / B),[\phi]$ belongs to $P(A / B)^{(G)}$ if and only if, for any $\sigma$ 
in $G$, there is a $B$ - $B$-isomorphism $f_{\sigma}: P \rightarrow J_{\sigma} \otimes{ }_{B} P \otimes{ }_{B} J_{\sigma-1}$ such that the diagram

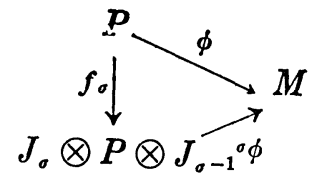

is commutative, where $\left({ }^{\circ} \phi\right)\left(x_{\sigma} \otimes p \otimes x_{\sigma}^{\prime}\right)=x_{\sigma} \cdot \phi(p) x_{\sigma}^{\prime}$. Here we shall check that $P(A / B)^{(G)}$ is closed with respect to inverse. We may assume that $P \subseteq M$ and $P^{*} \subseteq M^{*}$ (cf. [19; Lemma 3.1]). Then $P^{*}=\left\{g \in M^{*} \mid P^{g}\right.$ $\subseteq B\}$. In this sense, $(P) J_{\sigma} P^{*} J_{\sigma-1}=\left(P J_{\sigma}\right) P^{*} J_{\sigma-1}=\left(J_{\sigma} P\right) P^{*} J_{\sigma-1}=J_{\sigma}\left((P) P^{*}\right) J_{\sigma-1}$ $=J_{\sigma} J_{\sigma-1}=B$, and so $J_{\sigma} P^{*} J_{\sigma-1} \subseteq P^{*}$ for all $\sigma \in G$. Hence $J_{\sigma} P^{*} J_{o-1}=P^{*}$ for all $\sigma \in G$.

We put $P_{K}(A / B)^{(G)}=P_{K}(A / B) \cap P(A / B)^{(G)}$. Further we define Aut $(A / B)^{(G)}=\left\{f \in \operatorname{Aut}(A / B) \mid f\left(J_{\sigma}\right)=J_{\sigma}\right.$ for all $\left.\sigma \in G\right\}$. Then we have

Proposition 2.2. There is an exact sequence

$$
\begin{aligned}
1 \longrightarrow U(L) \cap U(K) \longrightarrow U(K) & \longrightarrow \operatorname{Aut}(A / B)^{(G)} \\
& \longrightarrow P_{K}(A / B)^{(G)} \longrightarrow \operatorname{Pic}_{K}(B)^{G} .
\end{aligned}
$$

Proof. The above sequence is a subsequence of the one in Th. 1.5. Therefore it suffices to prove that, for $f$ in Aut $(A / B)$, the image of $f$ is contained in $P_{K}(A / B)^{(G)}$ if and only if $f \in \operatorname{Aut}(A / B)^{(G)}$. However $J_{\sigma} \cdot B u_{f} J_{\sigma-1}=J_{\sigma} \cdot f\left(J_{\sigma}\right)^{-1} u_{f}$, so that $J_{\sigma} \cdot B u_{f} J_{\sigma-1}=B u_{f}$ if and only if $J_{\sigma} \cdot f\left(J_{\sigma}\right)^{-1}$ $=B$, or equivalently, $f\left(J_{o}\right)=J_{\sigma}$. This completes the proof.

Next we state several lemmas (which are well known).

For any two-sided $B$-module $U$, we denote by $V_{U}(B)\{u \in U \mid b u=u b$ for all $b \in B\}$.

Lemma 2.3. Let $B$ be an R-algebra, and $P$ an $R$-module such that ${ }_{R} P \mid{ }_{R} R$ (i.e., finitely generated and projective). Then $\operatorname{End}_{r}\left({ }_{B} B \otimes{ }_{R} P\right.$ ) $\stackrel{\approx}{\longrightarrow} B \otimes{ }_{R} \operatorname{End}_{r}\left({ }_{R} P\right)$ canonically, and $\left.{ }_{B} B \otimes{ }_{R} P_{B}\right|_{B} B_{B}$ (cf. [19]). And further $V_{B \otimes P}(B) \stackrel{\approx}{\longrightarrow} K \otimes_{R} P$ canonically, where $K$ is the center of $B$. Therefore if End $\left({ }_{R} P\right)=R$ then ${ }_{B} B \otimes{ }_{R} P_{B}$ is a Morita module.

Proof. The first assertion is well known. The remainder is evident, if ${ }_{R} P$ is free. Hence it is true for any $P$ such that $\left.{ }_{R} P\right|_{R} R$.

LeMmA 2.4. Let $\left.{ }_{B} M_{B}\right|_{B} B_{B}$. Then $M=B \cdot V_{M}(B) \stackrel{\approx}{\longrightarrow} B \otimes_{K} V_{M}(B)$ 
canonically, and $\left.{ }_{K} V_{M}(B)\right|_{K} K . \quad$ Further $\operatorname{End}_{r}\left({ }_{K} V_{M}(B)\right) \stackrel{\approx}{\longrightarrow} \operatorname{End}_{r}\left(_{B} M_{B}\right)$ and $\operatorname{End}_{r}\left({ }_{B} M\right) \stackrel{\approx}{\longrightarrow} B \otimes{ }_{K} \operatorname{End}_{r}\left({ }_{B} M_{B}\right)$, canonically.

Proof. $\left.{ }_{B} M_{B}\right|_{B} B_{B}$ implies that $V_{M}(K)=M$, and hence $M$ may be considered as a left $B^{e}$-module, where $B^{e}=B \otimes{ }_{K} B^{\circ p}$. Then $\left.{ }_{B e} M\right|_{B^{e}} B$. Evidently $\operatorname{Hom}_{r}\left({ }_{B e} B,{ }_{B e} M\right) \stackrel{\approx}{\longrightarrow} V_{M}(B)$ canonically. By [14; Th. 1.1], ${ }_{B e} M$ $\stackrel{\approx}{\longrightarrow} \operatorname{Hom}_{r}\left({ }_{B e} B^{e},{ }_{B e} M\right) \stackrel{\approx}{\longrightarrow} \operatorname{Hom}_{r}\left({ }_{B e} B^{e},{ }_{B e} B\right) \otimes{ }_{K} \operatorname{Hom}_{r}\left({ }_{B e} B,{ }_{B e} M\right) \stackrel{\approx}{\longrightarrow} B \otimes$ ${ }_{K} V_{M}(B),\left.{ }_{K} V_{M}\right|_{K} K$ and $\operatorname{End}_{r}\left({ }_{K} \operatorname{Hom}_{r}\left({ }_{B e} B,{ }_{B e} M\right)\right) \stackrel{\approx}{\longrightarrow} \operatorname{End}_{r}\left({ }_{B} M_{B}\right)$. Combining this with Lemma 2.3, we obtain the last assertion.

COROLlaRY 1. Further assume that $\operatorname{End}_{r}\left({ }_{B} M_{B}\right)=K$, Then ${ }_{B} M_{B}$ is a Morita module.

Corollary 2. Let $\left.{ }_{B} M_{B}\right|_{B} B_{B}$ and $\left.{ }_{B} M^{\prime}{ }_{B}\right|_{B} B_{B}$. Then ${ }_{B} M_{B} \stackrel{\approx}{\longrightarrow}{ }_{B} M^{\prime}{ }_{B}$ if and only if ${ }_{K} V_{M}(B) \stackrel{\approx}{\longrightarrow}{ }_{K} V_{M^{\prime}}(B)$.

The following corollary is repeatedly used to check commutativity of diagrams.

COROLlaRy 3. Let $\left.{ }_{B} M_{B}\right|_{B} B_{B}$ and $\left.{ }_{B} M^{\prime}{ }_{B}\right|_{B} B_{B}$. Then $V_{M \otimes M^{\prime}}(B) \stackrel{\approx}{\longrightarrow}$ $V_{M}(B) \otimes{ }_{K} V_{M}(B)$ canonically, and there is an isomorphism ${ }_{B} M \otimes M_{B}^{\prime} \rightarrow$ ${ }_{B} M^{\prime} \otimes M_{B}, m_{0} \otimes m^{\prime} \mapsto m^{\prime} \otimes m_{0}, m \otimes m_{0}^{\prime} \mapsto m_{0}^{\prime} \otimes m\left(m_{0} \in V_{M}(B), m \in M, m_{0}^{\prime} \in\right.$ $\left.V_{M^{\prime}}(B), m^{\prime} \in M^{\prime}\right)$, where unadorned $\otimes$ means $\otimes_{B}$. We call this isomorphism the "transposition" of $M$ and $M^{\prime}$

Proof. By Lemma 2.4, $M=B \otimes_{K} V_{M}(B)$ and $M^{\prime}=B \otimes_{K} V_{M^{\prime}}(B)$. Consequently, $M \otimes M^{\prime}=B \otimes{ }_{K} V_{M}(B) \otimes{ }_{K} V_{M^{\prime}}(B)$. Then, by Lemma 2.3, $V_{M \otimes M^{\prime}}(B) \stackrel{\approx}{\longrightarrow} V_{M}(B) \otimes_{K} V_{M^{\prime}}(B)$ canonically. Since $V_{M}(B) \otimes_{K} V_{M^{\prime}}(B) \stackrel{\approx}{\longrightarrow}$ $V_{M^{\prime}}(B) \otimes{ }_{K} V_{M}(B)$ by transposition, we obtain the latter assertion.

Remark. We put $\left\{[M] \in \operatorname{Pic}(B) \mid{ }_{B} M_{B} \sim{ }_{B} B_{B}\right\}=\operatorname{Pic}_{0}(B)$ ([19]). Then, by Lemma 2.3, Lemma 2.4, and Cor. 3 to Lemma 2.4, $\operatorname{Pic}_{K}(K) \stackrel{\approx}{\longrightarrow} \operatorname{Pic}_{0}(B)$, $[P] \mapsto\left[P \otimes{ }_{K} B\right]$.

The following lemma is also used to check commutativity of diagrams

LEMMA 2.5. Let ${ }_{B} U \otimes{ }_{B} W_{B} \sim{ }_{B} B_{B} \sim{ }_{B} M_{B}$. If $x \in V_{M}(B)$ and $\sum_{i} u_{i}$ $\otimes w_{i} \in V_{U \otimes W}(B)$, then $\sum_{i} u_{i} \otimes x \otimes w_{i} \in V_{U \otimes M \otimes W}(B)$.

Proof. For any $x$ in $V_{M}(B), U \otimes{ }_{B} W \rightarrow U \otimes M \otimes W, u \otimes w \mapsto u \otimes x \otimes w$ is a $B$ - $B$-homomorphism.

Next we shall define an abelian group $C(\Delta / B)$, which is the main object in the present paper. In the rest of this section, unadorned $\otimes$ 
always means $\otimes_{B} . \quad C(\Delta / B)$ consists of all isomorphic classes of generalized crossed products $\oplus_{\sigma \in G} V_{\sigma} / B$ of $B$ with $G$ such that ${ }_{B} V_{\sigma B} \sim{ }_{B} J_{\sigma B}$ for all $\sigma \in G$ (cf. [19]). Let $\oplus V_{\sigma} / B$ and $\oplus W_{\sigma} / B$ be generalized crossed products of $B$ with $G$, and let $f$ be a $B$-ring isomorphism from $\oplus V_{\sigma} / B$ to $\oplus W_{\sigma} / B$. If $f\left(V_{\sigma}\right)=W_{\sigma}$ for all $\sigma \in G$, we call $f$ an isomorphism as generalized crossed products. Precisely a generalized crossed product $\oplus V_{\sigma} / B$ is written as $\left(\oplus V_{\sigma} / B, f_{\sigma, \tau}\right)$, and its isomorphic class is denoted by $\left[\oplus V_{\sigma} / B, f_{\sigma, \tau}\right]$, where $f_{\sigma, \tau}: V_{\sigma} \otimes V_{\tau} \rightarrow V_{\sigma \tau}$ is the multiplication. In particular, the multiplication of $\Delta$ is denoted by $\phi_{\sigma, \tau}$. However we denote often $\left(\oplus J_{\sigma} / B, \phi_{\sigma, \tau}\right)$ by $\oplus J_{\sigma} / B$, simply. Let $\left(\oplus V_{\sigma} / B, f_{\sigma, \tau}\right)$ and $\left(\oplus W_{\sigma} / B, g_{\sigma, \tau}\right)$ be generalized crossed products in $C(\Delta / B)$. Then the $\sigma$-component of the product of $\left(\oplus V_{\sigma} / B, f_{\sigma, \tau}\right)$ and $\left(\oplus W_{\sigma} / B, g_{\sigma, \tau}\right)$ is defined as $V_{\sigma} \otimes J_{\sigma-1} \otimes W_{\sigma}$. The multiplication is defined by $h_{\sigma, \tau}: V_{\sigma} \otimes J_{\sigma^{-1}} \otimes W_{\sigma} \otimes V_{\tau} \otimes J_{\tau^{-1}} \otimes W_{\tau} \stackrel{t}{\longrightarrow} V_{\sigma} \otimes V_{\tau}$ $\otimes J_{\tau^{-1}} \otimes J_{\sigma^{-1}} \otimes W_{\sigma} \otimes W_{\tau} \stackrel{*}{\longrightarrow} V_{\sigma \tau} \otimes J_{(\sigma \tau)-1} \otimes W_{\sigma \tau}$, where $t$ is the transposition of $J_{\sigma-1} \otimes W_{\sigma}$ and $V_{\tau} \otimes J_{\tau-1}$, and $*=f_{\sigma, \tau} \otimes \phi_{\sigma, \tau} \otimes g_{\sigma, \tau}$. The associativity of the above multiplication is proved by making use of Cor. 3 to Lemma 2.4. If we identify the canonical isomorphism $B \otimes B \otimes B \rightarrow B$, then we have a generalized crossed product $\left(\oplus\left(V_{\sigma} \otimes J_{\sigma-1} \otimes W_{\sigma}\right) / B, h_{\sigma, \tau}\right)$. The associativity of this composition in $C(\Delta / B)$ is proved by using Cor. 3 to Lemma 2.4, too. Evidently $\left[\oplus J_{\sigma} / B, \phi_{\sigma, \tau}\right]$ is the identity element of $C(\Delta / B)$. The $\sigma$-component of the inverse of $\left(\oplus V_{\sigma} / B, f_{\sigma, \tau}\right)$ is $J_{\sigma} \otimes V_{\sigma}^{*} \otimes J_{\sigma}$, where $V_{\sigma}^{*}=\operatorname{Hom}_{r}\left({ }_{B} V_{\sigma},{ }_{B} B\right)$. The multiplication is defined by $f_{\sigma, \tau}^{*}: J_{\sigma} \otimes$ $\left(V_{\sigma}^{*} \otimes J_{\sigma}\right) \otimes\left(J_{\tau} \otimes V_{\tau}^{*}\right) \otimes J_{\tau} \stackrel{t}{\longrightarrow} J_{\sigma} \otimes\left(J_{\tau} \otimes V_{\tau}^{*}\right) \otimes\left(V_{\sigma}^{*} \otimes J_{\sigma}\right) \otimes J_{\tau} \stackrel{\phi \otimes * \otimes \phi}{\longrightarrow} J_{\sigma \tau} \otimes$ $V_{o \tau}^{*} \otimes J_{\sigma \tau}$, where $*: V_{\tau}^{*} \otimes V_{\sigma}^{*} \rightarrow\left(V_{\sigma} \otimes V_{\tau}\right)^{*} \rightarrow V_{\sigma \tau}^{*}$ is the canonical isomorphism induced by $f_{\sigma, \tau}$. We identify the canonical isomorphism $B \otimes B^{*}$ $\otimes B \rightarrow B$, and we have a generalized crossed product $\left(\oplus\left(J_{\sigma} \otimes V_{\sigma}^{*} \otimes J_{\sigma}\right) / B\right.$, $\left.f_{\sigma, \tau}^{*}\right)$. By the isomorphism $V_{\sigma} \otimes\left(J_{\sigma-1} \otimes J_{\sigma}\right) \otimes V_{\sigma}^{*} \otimes J_{\sigma} \rightarrow\left(V_{\sigma} \otimes V_{\sigma}^{*}\right) \otimes J_{\sigma} \rightarrow$ $J_{\sigma}$, the product of $\left(\oplus V_{\sigma} / B, f_{\sigma, \tau}\right)$ and $\left(\oplus\left(J_{\sigma} \otimes V_{\sigma}^{*} \otimes J_{\sigma}\right) / B, f_{\sigma, \tau}^{*}\right)$ is isomorphic to $A$, as generalized crossed products. Hence $C(\Delta / B)$ is a group. Finally $C(\Delta / B)$ is an abelian group, because the isomorphism $V_{\sigma} \otimes J_{\sigma-1}$ $\otimes W_{\sigma} \rightarrow V_{\sigma} \otimes J_{\sigma-1} \otimes W_{\sigma} \otimes\left(J_{\sigma-1} \otimes J_{\sigma}\right) \stackrel{t}{\longrightarrow} W_{\sigma} \otimes J_{\sigma-1} \otimes V_{\sigma} \otimes\left(J_{\sigma-1} \otimes J_{\sigma}\right) \rightarrow W_{\sigma}$ $\otimes J_{\sigma-1} \otimes V_{\sigma}$ is an isomorphism as generalized crossed products, where $t$ is the transposition of $V_{\sigma} \otimes J_{\sigma-1}$ and $W_{\sigma} \otimes J_{\sigma-1}$. By $C_{0}(\Delta / B)$, we denote the subgroup of all generalized crossed products $\left[\oplus V_{\sigma} / B, f_{\sigma, \tau}\right]$ such that ${ }_{B} V_{\sigma_{B}} \stackrel{\approx}{\longrightarrow}{ }_{B} J_{\sigma_{B}}$ for all $\sigma \in G$. We put $\operatorname{Pic}_{K}(B)^{[G]}=\left\{\left.[P] \in \operatorname{Pic}_{K}(B)\right|_{B} P \otimes J_{\sigma}\right.$ $\otimes * P_{B} \sim{ }_{B} J_{\sigma_{B}}$ for all $\sigma$ in $\left.G\right\}$, where $* P=\operatorname{Hom}_{l}\left(P_{B}, B_{B}\right)$, and " $\sim$ " means 
"similar" (cf. [19]). Then $\operatorname{Pic}_{K}(B)^{[G]}$ is evidently a subgroup of $\operatorname{Pic}_{K}(B)$. Then the canonical isomorphism $* P \otimes P \rightarrow B$ induces an isomorphism $P \otimes$ $J_{\sigma} \otimes\left({ }^{*} P \otimes P\right) \otimes J_{\tau} \otimes * P \rightarrow P \otimes J_{\sigma} \otimes J_{\tau} \otimes * P$, and we obtain ${ }^{P} \phi_{\sigma, \tau}:\left(P \otimes J_{\sigma}\right.$ $\otimes * P) \otimes\left(P \otimes J_{\tau} \otimes * P\right) \rightarrow P \otimes J_{\sigma} \otimes J_{\tau} \otimes * P \stackrel{|\otimes \phi \otimes|}{\longrightarrow} P \otimes J_{o \tau} \otimes * P . \quad$ Then $\left(\oplus\left(P \otimes J_{\sigma} \otimes * P\right) / B,{ }^{P} \phi_{\sigma, \tau}\right)$ is a generalized crossed product, and $[P] \mapsto$ $\left[\oplus\left(P \otimes J_{\sigma} \otimes * P\right) / B,{ }^{P} \phi_{\sigma, \tau}\right]$ is a group homomorphism from $\mathrm{Pic}_{K}(B)^{[G]}$ to $C(\Delta / B)$. Thus we have proved the following theorem

THEOREM 2.6. $C(\Delta / B)$ is an abelian group with identity $\Delta / B$, and $C_{0}(\Delta / B)$ is a subgroup of $C(\Delta / B)$. There is a commutative diagram

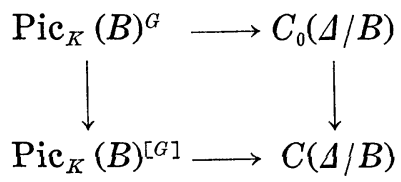

Remark. $C_{0}(\Delta / B)$ is isomorphic to $H^{2}(G, U(K))$. The isomorphism is defined as follows: Let $\left[\oplus J_{\sigma} / B, f_{\sigma, \tau}\right]$ be in $C_{0}(\Delta / B)$. Then, for any $\sigma, \tau$ in $G$, there exists uniquely $a_{\sigma, \tau} \in U(K)$ such that $f_{\sigma, \tau}\left(x_{\sigma} \otimes x_{\tau}\right)=$ $a_{\sigma, \tau} \cdot \phi_{\sigma, \tau}\left(x_{\sigma} \otimes x_{\tau}\right)$ for all $x_{\sigma} \in J_{\sigma}, x_{\tau} \in J_{\tau}$. Then $\left\{a_{\sigma, \tau} \mid \sigma, \tau \in G\right\}$ is a (normalized) factor set, and $\left[\oplus J_{\sigma} / B, f_{\sigma, \tau}\right] \mapsto$ class $\left\{a_{\sigma, \tau}\right\}$ is an isomorphism. $\left(\oplus J_{\sigma} / B\right.$, $\left.f_{\sigma, \tau}\right)$ may be written as $\left(\oplus J_{\sigma} / B, a_{\sigma, \tau}\right)$ when $\Delta$ is fixed.

Proposition 2.7. There is an exact sequence

$$
P_{K}(\Delta / B)^{\langle G)} \longrightarrow \operatorname{Pic}_{K}(B)^{G} \longrightarrow C_{0}(\Delta / B)
$$

Proof. The semi-exactness follows from the definition of $P_{K}(\Delta / B)^{(G)}$ $([19 ; \S 3])$. Let $[P] \in \mathrm{Pic}_{K}(B)^{G}$ be in the kernel. Then $\left(\oplus\left(P \otimes J_{\sigma} \otimes * P\right)\right.$, $\left.{ }^{P} \phi_{\sigma, \tau}\right)$ is isomorphic to $\left(\oplus J_{\sigma}, \phi_{\sigma, \tau}\right)=\Delta$. However, by [19; p. 116], $(\oplus P \otimes$ $\left.\left.J_{\sigma} \otimes{ }^{*} P\right),{ }^{P} \phi_{\sigma, \tau}\right) / B$ is isomorphic to $\operatorname{End}_{l}\left(P \otimes{ }_{B} \Delta_{4}\right) / B$, as rings, and so we have a Morita module ${ }_{\Delta} P \otimes{ }_{B} \Delta_{\Delta}$. Then the canonical homomorphism $P$ to $P \otimes \Delta, p \mapsto p \otimes 1$ is in $P_{K}(\Delta / B)^{(G)}$.

An abelian group $B(\Delta / B)$ is defined by the following exact sequence:

$$
\operatorname{Pic}_{K}(B)^{[G]} \longrightarrow C(\Delta / B) \longrightarrow B(\Delta / B) \longrightarrow 1
$$

Then we have

Proposition 2.8. There is an exact sequence

$$
\mathrm{Pic}_{K}(B)^{G} \longrightarrow C_{0}(\Delta / B) \longrightarrow B(\Delta / B)
$$


Proof. The semi-exactness is trivial. If $\left[\oplus J_{\sigma}, f_{\sigma, \tau}\right]$ is in the kernel of $C_{0}(\Delta / B) \rightarrow B(\Delta / B)$, then there is $[P]$ in $\mathrm{Pic}_{K}(B)^{[G]}$ such that $[P] \mapsto$ $\left[\oplus J_{o}, f_{v, \tau}\right]$ under the homomorphism $\operatorname{Pic}_{K}(B)^{[G]} \rightarrow C(\Delta / B)$. Then it is evident that $[P]$ is in $\mathrm{Pic}_{K}(B)^{G}$.

By Remark to Cor. 3 to-Lemma 2.4, $\operatorname{Pic}_{K}(K) \rightarrow \operatorname{Pic}_{0}(B),\left[P_{0}\right] \mapsto$ $\left[P_{0} \otimes{ }_{K} B\right]$ is an isomorphism, and $[P] \mapsto\left[V_{P}(B)\right]$ is its inverse.

Proposition 2.9. The above isomorphism is a G-isomorphism.

Proof. Let $[P]$ be in $\mathrm{Pic}_{0}(B)$. Then $P=B \otimes{ }_{K} V_{P}(B)$, and $J_{\sigma} \otimes P$ $\otimes J_{\sigma-1} \stackrel{\approx}{\longrightarrow} J_{\sigma} \otimes\left(B \otimes{ }_{K} V_{P}(B)\right) \otimes J_{\sigma-1} \stackrel{\approx}{\longrightarrow}\left(J_{\sigma} \otimes{ }_{K} V_{P}(B)\right) \otimes J_{\sigma-1}$ as two-sided $B$-modules. It is easily seen that $J_{\sigma} \otimes{ }_{K} V_{P}(B) \rightarrow K u_{\sigma} \otimes{ }_{K} V_{P}(B) \otimes{ }_{K} K u_{\sigma-1}$ $\otimes{ }_{K} J_{\sigma}, x_{\sigma} \otimes p_{0} \mapsto u_{\sigma} \otimes p_{0} \otimes u_{\sigma-1} \otimes x_{\sigma}$ is a $B$ - $B$-isomorphism, where $\sigma$ denotes the automorphism induced by $J_{\sigma}$. Therefore $J_{\sigma} \otimes P \otimes J_{\sigma-1} \stackrel{\approx}{\longrightarrow} K u_{\sigma} \otimes$ ${ }_{K} V_{P}(B) \otimes{ }_{K} K u_{\sigma-1} \otimes{ }_{K} B, x_{\sigma} \otimes p_{0} \otimes x_{\sigma-1} \mapsto u_{\sigma} \otimes p_{0} \otimes u_{\sigma-1} \otimes x_{\sigma} x_{\sigma-1}\left(x_{\sigma} \in J_{\sigma}, x_{\sigma-1} \in\right.$ $\left.J_{\sigma^{-1}}, p_{0} \in V_{P}(B)\right)$ is a $B$-B-isomorphism. Hence, by Lemma 2.3, $V_{J o \otimes P \otimes J \sigma^{-1}}(B) \stackrel{\approx}{\longrightarrow} K u_{\sigma} \otimes_{K} V_{P}(B) \otimes{ }_{K} K u_{\sigma-1}$, as $K$-modules. This completes the proof.

Corollary. $Z^{1}\left(G, \operatorname{Pic}_{K}(K)\right) \stackrel{\approx}{\longrightarrow} Z^{1}\left(G, \operatorname{Pic}_{0}(B)\right)$.

There is a group homomorphism $\left[\oplus V_{\sigma}, f_{\sigma, \tau}\right] \mapsto\left(\sigma \rightarrow\left[V_{\sigma}\right]\left[J_{\sigma}\right]^{-1}\right)(\sigma \in G)$ from $C(\Delta / B)$ to $Z^{1}\left(G, \mathrm{Pic}_{0}(B)\right)$. Then the following sequence is exact:

$$
1 \longrightarrow C_{0}(\Delta / B) \longrightarrow C(\Delta / B) \longrightarrow Z^{1}\left(G, \operatorname{Pic}_{0}(B)\right)
$$

$\bar{H}^{1}\left(G, \mathrm{Pic}_{0}(B)\right)$ is defined by the exactness of the following row:

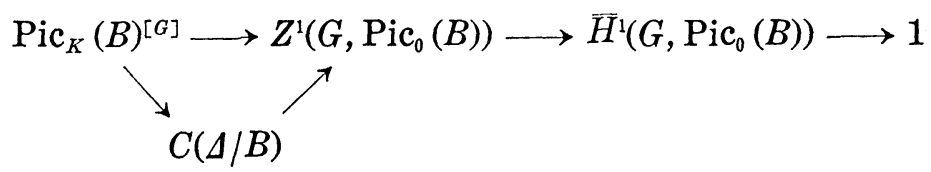

Proposition 2.10. $\quad C_{0}(\Delta / B) \rightarrow B(\Delta / B) \rightarrow \bar{H}^{1}\left(G, \mathrm{Pic}_{0}(B)\right)$ is exact.

Proof. Evidently the above sequence is semi-exact. Let $\left[\left[\oplus V_{\sigma}, f_{\sigma, \tau}\right]\right]$ (the class of $\left[\oplus V_{\sigma}, f_{\sigma, \tau}\right]$ in $B(\Delta / B)$ ) be in the kernel. Then there is a $[P] \in \mathrm{Pic}_{K}(B)^{[G]}$ such that $P \otimes J_{\sigma} \otimes * P \stackrel{*}{\longrightarrow} V_{\sigma}$ for all $\sigma \in G$, where ${ }^{*} P=$ $\operatorname{Hom}_{l}\left(P_{B}, B_{B}\right)$. For any $\sigma \in G$, we fix an isomorphism $h_{\sigma}: P \otimes J_{\sigma} \otimes * P$ $\rightarrow V_{\sigma} \cdot f_{\sigma, \tau}^{\prime}$ is defined by the commutativity of the diagram 


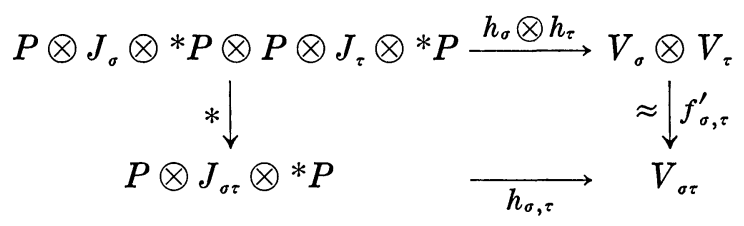

where $*$ is defined by $* P \otimes P \stackrel{\approx}{\longrightarrow} B$ (canonical) and $\phi_{\sigma, \tau}$. Then $\left(\oplus V_{\sigma}\right.$, $\left.f_{\sigma, \tau}^{\prime}\right)$ differs from $\left(\oplus V_{\sigma}, f_{\sigma, \tau}\right)$ by some factor set $\left\{a_{\sigma, \tau}\right\}$, i.e., $f_{\sigma, \tau}^{\prime}=a_{\sigma, \tau} f_{\sigma, \tau}$ (cf. Remark to Th. 2.6.). Then, by the canonical isomorphism $J_{\sigma} \otimes J_{\sigma-1}$ $\otimes V_{\sigma} \stackrel{\approx}{\longrightarrow} V_{\sigma},\left(\oplus J_{\sigma}, a_{\sigma, \tau}\right) \times\left(\oplus V_{\sigma}, f_{\sigma, \tau}\right)$ is isomorphic to $\left(\oplus V_{\sigma}, f_{\sigma, \tau}^{\prime}\right)$. Since $\left(\oplus V_{\sigma}, f_{\sigma, \tau}^{\prime}\right)$ is isomorphic to $\left(\oplus\left(P \otimes J_{\sigma} \otimes * P\right),{ }^{P} \phi_{\sigma, \tau}\right)$, this completes the proof.

Proposition 2.11. There is an exact sequence

$$
B(\Delta / B) \longrightarrow \bar{H}^{1}\left(G, \operatorname{Pic}_{0}(B)\right) \longrightarrow H^{3}(G, U(K)) .
$$

Proof. For $\phi$ in $Z^{1}\left(G, \operatorname{Pic}_{0}(B)\right)$, a homomorphism $\Phi$ from $G$ to $\operatorname{Pic}(B)$ is defined by $\Phi(\sigma)=\phi(\sigma)\left[J_{\sigma}\right]$. Let $\Phi(\sigma)=\left[U_{\sigma}\right]$ and $U_{1}=B$. Then $U_{\sigma} \sim J_{\sigma}$, as $B$ - $B$-modules, for all $\sigma \in G$. For $\sigma, \tau$ in $G$, we take a $B$ - $B$-isomorphism $f_{\sigma, \tau}: U_{\sigma} \otimes U_{\tau} \rightarrow U_{\sigma \tau}$. If $\sigma=1$ or $\tau=1$ then we take $f_{\sigma, \tau}$ as a canonical one. Then, for any $\sigma, \tau, \gamma$ in $G$, there exists uniquely $u(\sigma, \tau, \gamma) \in$ $U(K)$ such that $u(\sigma, \tau, \gamma) f_{\sigma, \tau \tau}\left(I_{\sigma} \otimes f_{\tau, \gamma}\right)(x)=f_{\sigma \tau, \gamma}\left(f_{\sigma, \tau} \otimes I_{\gamma}\right)(x)$ for all $x$ in $J_{\sigma \tau \gamma}$, where $I_{\sigma}$ is the identity of $U_{\sigma}$.

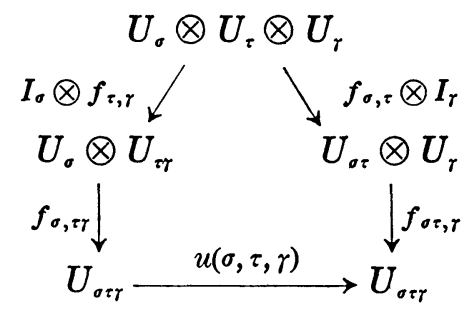

If $\sigma=1$ or $\tau=1$ or $\gamma=1$, then $u(\sigma, \tau, \gamma)=1$. Let $f_{\sigma, \tau}^{\prime}$ be another isomorphism from $U_{\sigma} \otimes U_{\tau}$ to $U_{\sigma \tau}$, and let $u^{\prime}(\sigma, \tau, \gamma)$ be the one determined by $f_{\sigma, \tau}^{\prime}$. Then, for any $\sigma, \tau$ in $G$, there exists a unique $u(\sigma, \tau) \in U(K)$ such that $u(\sigma, \tau) f_{\sigma, \tau}=f_{\sigma, \tau}^{\prime}$. If $\sigma=1$ or $\tau=1$, then $u(\sigma, \tau)=1$. It is easily seen that $u^{\prime}(\sigma, \tau, \gamma)=u(\sigma \tau, \gamma) u(\sigma, \tau) \cdot{ }^{\sigma} u(\tau, \gamma)^{-1} u(\sigma, \tau \gamma)^{-1} u(\sigma, \tau, \gamma)$. Let $H$ be the group of all functions $u$ from $G \times G \times G$ to $U(K)$. Then $Z^{1}\left(G, \operatorname{Pic}_{0}(B)\right)$ $\rightarrow H / B^{3}(G, U(K)), \phi \mapsto$ class $\{u(\sigma, \tau, \gamma)\}$ is well defined, and this induces $\alpha: \bar{H}^{1}\left(G, \operatorname{Pic}_{0}(B)\right) \rightarrow H / B^{3}(G, U(K))$, where $B^{3}(G, U(K))$ consists of all $u(-,-,-) \in H$ such that $u(\sigma, \tau, \gamma)=u(\sigma \tau, \gamma) u(\sigma, \tau) \cdot{ }^{\sigma} u(\tau, \gamma)^{-1} u(\sigma, \tau \gamma)^{-1}$ for 
some mapping $u(-,-): G \times G \rightarrow U(K)$ such that $u(\sigma, \tau)=1$ provided $\sigma=1$ or $\tau=1$. If class $\{u(\sigma, \tau, \gamma)\}=1$ then, for a suitable choice of $f_{\sigma, \tau}$, we can take $u(\sigma, \tau, \gamma)=1$ for all $\sigma, \tau, \gamma \in G$. Next we shall show that $\alpha$ is a homomorphism from $\bar{H}^{1}\left(G, \mathrm{Pic}_{0}(B)\right)$ to $H / B^{3}(G, U(K))$. We take another $\psi \in Z^{1}\left(G, \operatorname{Pic}_{0}(B)\right)$, and put $\Psi(\sigma)=\psi(\sigma)\left[J_{\sigma}\right]=\left[W_{\sigma}\right]$. And let each $g_{\sigma, \tau}: W_{\sigma} \otimes W_{\tau} \rightarrow W_{\sigma \tau}$ be a $B$-B-isomorphism, and $u_{1}(\sigma, \tau, \gamma)$ be the one determined by $g_{\sigma, \tau^{*}}$ Put $\phi \psi=\pi$. Then $\Pi(\sigma)=\phi(\sigma) \psi(\sigma)\left[J_{\sigma}\right]=\phi(\sigma)\left[J_{\sigma}\right]\left[J_{\sigma}\right]^{-1}$ - $\psi(\sigma)\left[J_{\sigma}\right]=\Phi(\sigma)\left[J_{\sigma}\right]^{-1} \psi(\sigma)=\left[U_{\sigma} \otimes J_{\sigma-1} \otimes W_{\sigma}\right]$. We take an isomorphism $k_{\sigma, \tau}: U_{\sigma} \otimes J_{\sigma^{-1}} \otimes W_{\sigma} \otimes U_{\tau} \otimes J_{\tau^{-1}} \otimes W_{\tau} \stackrel{t}{\longrightarrow} U_{\sigma} \otimes U_{\tau} \otimes J_{\tau^{-1}} \otimes J_{\sigma-1} \otimes W_{\sigma} \otimes W_{\tau}$ $\stackrel{*}{\longrightarrow} U_{\sigma \tau} \otimes J_{(\sigma \tau)-1} \otimes W_{\sigma \tau}$, where $t$ is the transposition of $J_{\sigma-1} \otimes W_{\sigma}$ and $U_{\tau} \otimes J_{\tau-1}$, and $*=f_{\sigma, \tau} \otimes \phi_{\tau^{-1, \sigma-1}} \otimes g_{\sigma, \tau}$. Then, by using of Cor. 3 to Lemma 2.4 , it is easily seen that $u(\sigma, \tau, \gamma) u_{1}(\sigma, \tau, \gamma) k_{\sigma, \tau \gamma}\left(I_{\sigma} \otimes k_{\tau, \gamma}\right)=k_{\sigma \tau, \gamma}\left(k_{\sigma, \tau} \otimes I_{\gamma}\right)$. The fact that $\operatorname{Im} \alpha$ is contained in $H^{3}(G, U(K))$ will be proved later. Thus we have obtained the following theorem, which may be considered as a generalization of Chase, Harrison, Resenberg [8; Cor. 5.5].

THEOREM 2.12. Let $G$ be a group, and $\Delta / B=\left(\oplus J_{\sigma}, \phi_{\sigma, \tau}\right)$ be a generalized crossed product of $B$ with $G$. Let $C$ and $K$ be the centers of $\Delta$ and $B$, respectively. Then there is an exact sequence

$$
\begin{aligned}
1 \longrightarrow U(C) \cap U(K) \longrightarrow U(K) \longrightarrow \operatorname{Aut}(\Delta / B)^{(G)} \\
\longrightarrow P_{K}(\Delta / B)^{(G)} \longrightarrow \operatorname{Pic}_{K}(B)^{G} \longrightarrow C_{0}(\Delta / B) \\
\longrightarrow \bar{H}^{1}\left(G, \operatorname{Pic}_{0}(B)\right) \longrightarrow H^{3}(G, U(K)) .
\end{aligned}
$$

Proof. This follows from Propositions 2.2, 2.7, 2.8, 2.10 and 2.11.

Remark. The above sequence can be expressed as a seven term exact sequence:

$$
\begin{aligned}
& 1 \longrightarrow H^{1}(G, U(K)) \longrightarrow P_{K}(\Delta / B)^{(G)} \longrightarrow \operatorname{Pic}_{K}(B)^{G} \longrightarrow H^{2}(G, U(K)) \\
& \longrightarrow B(\Delta / B) \longrightarrow \bar{H}^{1}\left(G, \operatorname{Pic}_{0}(B)\right) \longrightarrow H^{3}(G, U(K)) .
\end{aligned}
$$

In fact, for any $f \in \operatorname{Aut}(\Delta / B)^{(G)}$ and any $\sigma \in G$, there exists uniquely $c_{0} \in U(K)$ such that $f\left(x_{\sigma}\right)=c_{\sigma} x_{\sigma}$ for all $x_{\sigma} \in J_{\sigma}$. Then it is easily seen that $c_{\sigma \tau}=c_{\sigma} \cdot{ }^{\sigma} c_{\tau}$ for all $\sigma, \tau \in G$, and we have an isomorphism $\operatorname{Aut}(\Delta / B)^{(G)}$ $\stackrel{\approx}{\rightarrow} Z^{1}(G, U(K))$. Evidently the image of $U(K)$ in Aut $(\Delta / B)^{(G)}$ corresponds to $B^{1}(G, U(K))$.

Let $P_{\sigma}(\sigma \in G)$ be a family of Morita $B$-B-modules such that ${ }_{B} P_{\sigma B}$ $\sim{ }_{B} B_{B}, P_{1}=B$. Then ${ }_{B} P_{\sigma} \otimes J_{\sigma B} \sim{ }_{B} J_{\sigma B}$. Put $V_{P_{\sigma}}(B)=P_{0, \sigma}$. Then ${ }_{K} P_{0, \sigma}$ 
$\sim{ }_{K} K$, and so ${ }_{K} P_{0, \sigma} \otimes{ }_{K} K u_{\sigma_{K}} \sim{ }_{K} K u_{\sigma_{K}}$. It was noted in the proof of Prop. 2.9 that $K u_{\sigma} \otimes{ }_{K} P_{0, \tau} \otimes{ }_{K} K u_{\sigma-1} \stackrel{\approx}{\longrightarrow} V_{J \sigma \otimes P r \otimes J \sigma-1}(B)$, as $K$-K-modules, $u_{\sigma} \otimes p_{\tau} \otimes u_{\sigma-1} \mapsto \sum_{i} a_{i} \otimes p_{\tau} \otimes a_{i}^{\prime}$, where $a_{i} \in J_{\sigma}, a_{i}^{\prime} \in J_{\sigma-1}, \sum_{i} a_{i} a_{i}^{\prime}=1$. Let $f_{\sigma, \tau}^{*}: P_{\sigma} \otimes J_{\sigma} \otimes P_{\tau} \otimes J_{\sigma-1} \rightarrow P_{o \tau}(\sigma, \tau \in G)$ be a family of $B$-B-isomorphisms. Then, since $V_{J \sigma \otimes P \tau \otimes J \sigma-1}(B) \stackrel{\approx}{\longrightarrow} K u_{\sigma} \otimes_{K} P_{0, \tau} \otimes_{K} K u_{\sigma-1}$, each $f_{\sigma, \tau}^{*}$ induces a $K$ - $K$-isomorphism $f_{0, \sigma, \tau}^{*}: P_{0, \sigma} \otimes{ }_{K} K u_{\sigma} \otimes_{K} P_{0, \tau} \otimes{ }_{K} K u_{\sigma-1} \rightarrow P_{0, \sigma \tau}$ (cf. Cor. 3 to Lemma 2.4), and conversely, and it is evident that $\left\{f_{\sigma, \tau}^{*} \mid \sigma, \tau \in G\right\} \mapsto$ $\left\{f_{0, \sigma, \tau}^{*} \mid \sigma, \tau \in G\right\}$ is a one to one mapping between them. This is nothing but an isomorphism in Cor. to Prop. 2.9, and we can prove the commutativity of the following diagram:

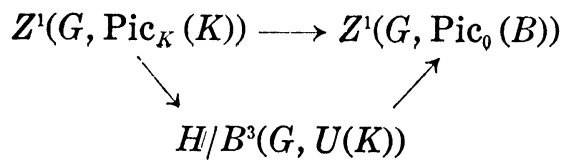

Then, by the same way as in [16; Lemma 8], the image of $Z^{1}\left(G, \mathrm{Pic}_{K}(K)\right)$ in $H / B^{3}(G, U(K))$ is contained in $H^{3}(G, U(K))$, and this completes the proof of Th. 2.12. On the other hand, $f_{\sigma, \tau}^{*}: P_{\sigma} \otimes J_{\sigma} \otimes P_{\tau} \otimes J_{\sigma-1} \stackrel{f_{\sigma, \tau}^{*} \otimes \phi_{\sigma, \tau}}{\longrightarrow}$ $P_{\sigma \tau}(\sigma, \tau \in G)$ induces $f_{\sigma, \tau}: P_{\sigma} \otimes J_{\sigma} \otimes P_{\tau} \otimes J_{\tau} \rightarrow\left(P_{\sigma} \otimes J_{\sigma} \otimes P_{\tau} \otimes J_{\sigma-1}\right) \otimes\left(J_{\sigma} \otimes J_{\tau}\right)$ $\rightarrow P_{\sigma \tau} \otimes J_{\sigma \tau}(\sigma, \tau \in G)$ and conversely, and $\left\{f_{\sigma, \tau}^{*} \mid \sigma, \tau \in G\right\} \mapsto\left\{f_{\sigma, \tau} \mid \sigma, \tau \in G\right\}$ is a 1-1 mapping. A similar fact holds with respect to $P_{0, \sigma}(\sigma \in G)$ and a crossed product $\oplus K u_{o}$ with trivial factor set: $\left\{f_{0, \sigma, \tau}^{*} \mid \sigma, \tau \in G\right\} \mapsto\left\{f_{0, \sigma, \tau} \mid \sigma, \tau \in G\right\}$. Let $\left\{f_{\sigma, \tau}\right\} \leftrightarrow\left\{f_{\sigma, \tau}^{*}\right\} \leftrightarrow\left\{f_{0, \sigma, \tau}^{*}\right\} \leftrightarrow\left\{f_{0, \sigma, \tau}\right\}$. Then $\left\{f_{\sigma, \tau}\right\}$ defines a generalized crossed product if and only if so is $\left\{f_{0, \sigma, \tau}\right\}$. Its proof is easy, but it is tedious, so we omit it. Next we shall show that $\left\{f_{\sigma, \tau}\right\} \mapsto\left\{f_{0, \sigma, \tau}\right\}$ is an isomorphism from $C(\Delta / B)$ to $C\left(\oplus K u_{\sigma} / K\right)$. To this end, let $\left[\oplus\left(Q_{\sigma} \otimes J_{\sigma}\right)\right.$, $\left.g_{\sigma, \tau}\right]$ be another element in $C(\Delta / B)$, and let $\left[\oplus\left(P_{\sigma} \otimes Q_{\sigma} \otimes J_{\sigma}\right), h_{\sigma, \tau}\right]$ be the product of $\left[\oplus\left(P_{\sigma} \otimes J_{\sigma}\right), f_{\sigma, \tau}\right]$ and $\left[\oplus\left(Q_{\sigma} \otimes J_{\sigma}\right), g_{\sigma, \tau}\right]$ (cf. the proof of Th. 2.6). Then $f_{\sigma, \tau}^{*}: P_{\sigma} \otimes J_{\sigma} \otimes P_{\tau} \otimes J_{\sigma-1} \stackrel{\approx}{\longrightarrow} P_{\sigma \tau}$ and $g_{\sigma, \tau}^{*}: Q_{\sigma} \otimes J_{\sigma} \otimes Q_{\tau} \otimes J_{\sigma-1}$ $\stackrel{\approx}{\longrightarrow} Q_{\sigma \tau}$ induce $f_{\sigma, \tau}^{*} \otimes g_{\sigma, \tau}^{*}: P_{\sigma} \otimes J_{\sigma} \otimes P_{\tau} \otimes J_{\sigma-1} \otimes Q_{\sigma} \otimes J_{\sigma} \otimes Q_{\tau} \otimes J_{\sigma-1} \stackrel{\approx}{\longrightarrow}$ $P_{\sigma \tau} \otimes Q_{\sigma \tau}$. Similarly $f_{0, \sigma, \tau}^{*}$ and $g_{0, \sigma, \tau}^{*}$ induce $f_{0, \sigma, \tau}^{*} \otimes g_{0, \sigma, \tau^{*}}^{*} \quad$ On the other hand there are isomorphisms $P_{\sigma} \otimes J_{\sigma} \otimes P_{\tau} \otimes J_{\sigma-1} \otimes Q_{\sigma} \otimes J_{\sigma} \otimes Q_{\tau} \otimes J_{\sigma-1}$ $\stackrel{t}{\longrightarrow} P_{\sigma} \otimes Q_{\sigma} \otimes J_{\sigma} \otimes P_{\tau} \otimes\left(J_{\sigma-1} \otimes J_{\sigma}\right) \otimes Q_{\tau} \otimes J_{\sigma-1} \stackrel{*}{\longrightarrow} P_{\sigma} \otimes Q_{\sigma} \otimes J_{\sigma} \otimes P_{\tau} \otimes Q_{\tau}$ $\otimes J_{\sigma-1}$, where $t$ is the transposition of $J_{\sigma} \otimes P_{\tau} \otimes J_{\sigma-1}$ and $Q_{\sigma}$. Similarly we have an isomorphism $P_{0, \sigma} \otimes K u_{\sigma} \otimes P_{0, \tau} \otimes K u_{\sigma-1} \otimes Q_{0, \sigma} \otimes K u_{\sigma} \otimes Q_{0, \tau} \otimes$ $K u_{\sigma-1} \rightarrow P_{0, \sigma} \otimes Q_{0, \sigma} \otimes K u_{\sigma} \otimes P_{0, \tau} \otimes Q_{0, \tau} \otimes K u_{\sigma-1}$ for all $\sigma, \tau \in G$. Then the following two diagrams are commutative: 
$P_{\sigma} \otimes J_{\sigma} \otimes P_{\tau} \otimes J_{\sigma-1} \otimes Q_{\sigma} \otimes J_{\sigma} \otimes Q_{\tau} \otimes J_{\sigma-1} \underset{f_{\sigma, \tau} \otimes g_{\sigma, \tau}^{*}}{\rightarrow} P_{\sigma \tau} \otimes Q_{\sigma \tau}$<smiles>[C+]1C=C1</smiles>

$P_{\sigma} \otimes Q_{\sigma} \otimes J_{\sigma} \otimes P_{\tau} \otimes Q_{\tau} \otimes J_{\sigma-1}$

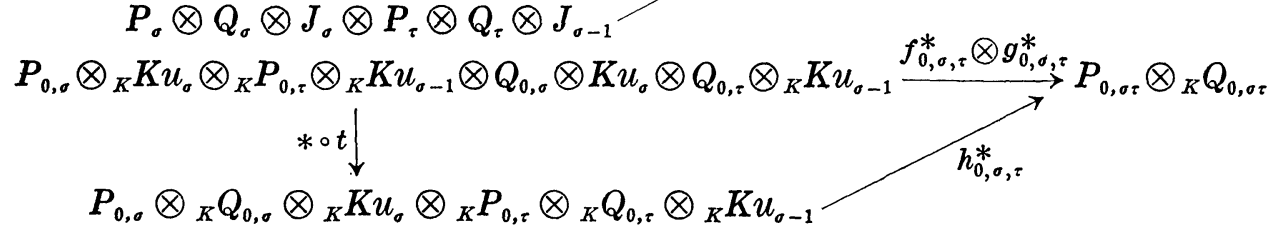

where $\left[\oplus\left(P_{0, \sigma} \otimes_{K} Q_{0, \sigma} \otimes_{K} K u_{\sigma}\right), h_{0, \sigma, \tau}\right]$ is the product of $\left[\oplus\left(P_{0, \sigma} \otimes{ }_{K} K u_{\sigma}\right), f_{0, \sigma, \tau}\right]$ and $\left[\oplus\left(Q_{0, \sigma} \otimes{ }_{K} K u_{\sigma}\right), g_{0, \sigma, \tau}\right]$. Then, since $\left\{f_{\sigma, \tau}^{*} \otimes g_{\sigma, \tau}^{*}\right\} \leftrightarrow\left\{f_{0, \sigma, \tau}^{*} \otimes g_{0, \sigma, \tau}^{*}\right\}$ is evident, we know that $\left\{h_{\sigma, \tau}\right\} \leftrightarrow\left\{h_{0, \sigma, \tau}\right\}$. Thus we have proved that $C(\Delta / B)$ $\rightarrow C\left(\oplus K u_{o} / K\right),\left\{f_{o, \tau}\right\} \mapsto\left\{f_{0, o, \tau}\right\}$ is an isomorphism. It is easily seen that $C_{0}(\Delta / B) \stackrel{\approx}{\longrightarrow} C_{0}\left(\oplus K u_{\sigma} / K\right)$ under the above isomorphism. Thus we have proved

THEOREM 2.13. There are commutative diagrams:

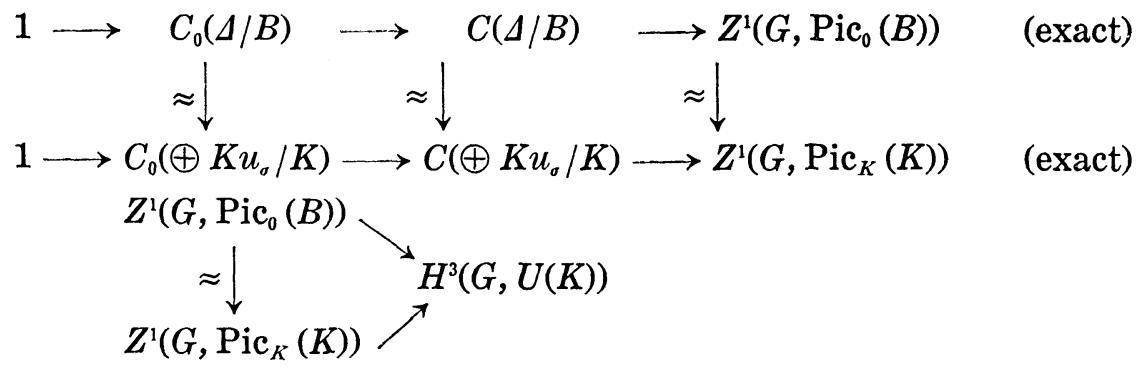

We shall further continue the study of the relation between $\Delta / B$ and $\oplus K u_{\sigma} / K$ (with trivial factor set).

Proposition 2.14. There exists a commutative diagram

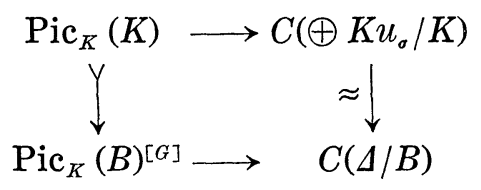

Proof. Let $\left[P_{0}\right] \in \operatorname{Pic}_{K}(k)$. It is necessary to prove that $\left(\oplus\left(P_{0} \otimes\right.\right.$ $\left.\left.{ }_{K} K u_{\sigma} \otimes{ }_{K} * P_{0}\right),{ }^{P_{0}} \phi_{0, \sigma, \tau}\right)$ corresponds to $\left(\oplus\left(\left(B \otimes{ }_{K} P_{0}\right) \otimes J_{\sigma} \otimes\left(B \otimes{ }_{K} * P_{0}\right)\right),{ }^{P} \phi_{\sigma, \tau}\right)$ under the isomorphism $C\left(\oplus K u_{\sigma} / K\right) \rightarrow C(\Delta / B)$, where $\phi_{0, \sigma, \tau}$ is the canonical isomorphism $K u_{\sigma} \otimes{ }_{K} K u_{\tau} \rightarrow K u_{\sigma \tau}, u_{\sigma} \otimes u_{\tau} \mapsto u_{\sigma \tau}, P=B \otimes{ }_{K} P_{0}$, and $* P_{0}=$ $\operatorname{Hom}_{l}\left(P_{0 K}, K_{K}\right)$ (cf. the proof of Th. 2.6). However this is done by using 
$K u_{\sigma} \otimes_{K} * P_{0} \otimes_{K} K u_{\sigma-1} \stackrel{\approx}{\longrightarrow} V_{J \sigma \otimes * P \otimes J \sigma-1}(B)$ and $* P \stackrel{\approx}{\longrightarrow} B \otimes_{K} * P_{0}$ canonically (cf. the proof of Th. 2.13).

Next we define a homomorphism from $P_{K}\left(\oplus K u_{\sigma} / K\right)^{(G)}$ to $P_{K}(\Delta / B)^{(G)}$. Let $\phi_{0}: P_{0} \rightarrow M_{0}$ be in $P_{K}\left(\oplus K u_{\sigma} / K\right)^{(G)}$. Then $K u_{\sigma} \otimes_{K} P_{0} \otimes{ }_{K} K u_{\sigma-1} \stackrel{\approx}{\longrightarrow}$ $V_{J \sigma \otimes P \otimes J \sigma-1}(B), \quad$ as $\quad K-K$-modules, $u_{\sigma} \otimes p_{0} \otimes u_{\sigma-1} \mapsto \sum_{i} a_{\sigma, i} \otimes\left(1 \otimes p_{0}\right) \otimes a_{\sigma, i}^{\prime}$, where $P=B \otimes{ }_{K} P_{0}, a_{\sigma, i} \in J_{\sigma}, a_{\sigma, i}^{\prime} \in J_{\sigma-1}, \sum_{i} a_{\sigma, i} a_{\sigma, i}^{\prime}=1$. Therefore $K u_{\sigma} \otimes$ ${ }_{K} P_{0} \otimes{ }_{K} K u_{\sigma-1} \otimes{ }_{K} J_{\sigma} \stackrel{\approx}{\longrightarrow} J_{\sigma} \otimes P, \quad$ as $\quad B$-B-modules, $\quad u_{\sigma} \otimes p_{0} \otimes u_{\sigma-1} \otimes x_{\sigma} \mapsto$ $x_{\sigma} \otimes\left(1 \otimes p_{0}\right)$ (cf. the proof of Prop. 2.9). Now, for the sake of simplicity, we may assume that $P_{0} \subseteq M_{0}$. Then $u_{\sigma} P_{0} u_{\sigma-1}=P_{0}$ for all $\sigma \in G$. Then $P_{0} \otimes{ }_{K} J_{\sigma} \stackrel{\approx}{\longrightarrow} J_{\sigma} \otimes{ }_{K} P_{0}$, as $B$-B-modules, $u_{\sigma} p_{0} u_{\sigma-1} \otimes x_{\sigma} \mapsto x_{\sigma} \otimes p_{0}$, and this induces a $B$ - $B$-isomorphism $P_{0} \otimes{ }_{K} \Delta(\stackrel{\approx}{\longrightarrow} P \otimes \Delta) \stackrel{\approx}{\longrightarrow} \Delta \otimes{ }_{K} P_{0}(\stackrel{\approx}{\longrightarrow} \Delta \otimes P)$. Then, by Lemma 1.2 , we have a Morita module ${ }_{\Delta} \Delta \otimes{ }_{K} P_{04}$, where $\left(x_{\sigma} \otimes p_{0}\right) x_{\tau}$ $=x_{\sigma} x_{\tau} \otimes u_{\tau-1} p_{0} u_{\tau}\left(x_{\sigma} \in J_{\sigma}, p_{0} \in P_{0}, x_{\tau} \in J_{\tau}\right)$. Hence the canonical homomorphism $\phi: B \otimes{ }_{K} P_{0}=P \rightarrow \Delta \otimes{ }_{K} P_{0}$ is in $P_{K}(\Delta / B)^{(G)}$. Let $\psi_{0}: Q_{0} \rightarrow U_{0}$ be another element of $P_{K}\left(\oplus K u_{\sigma} / K\right)^{(G)}$. Then $\left[\phi_{0}\right]\left[\psi_{0}\right]: P_{0} \otimes{ }_{K} Q_{0} \rightarrow M_{0} \otimes U_{0}$, $p_{0} \otimes q_{0} \mapsto \phi_{0}\left(p_{0}\right) \otimes \psi_{0}\left(q_{0}\right)$, where $\otimes^{\prime}$ means the tensor product over $\oplus K u_{o}$. On the other hand, [ $\phi][\psi]:\left(B \otimes{ }_{K} P_{0}\right) \otimes\left(B \otimes{ }_{K} Q_{0}\right) \rightarrow\left(\Delta \otimes{ }_{K} P_{0}\right) \otimes{ }_{\Delta}\left(\Delta \otimes_{K} Q_{0}\right)$ is the canonical map. Then it is easily seen that the canonical isomorphism $\Delta \otimes{ }_{K} P_{0} \otimes{ }_{K} Q_{0} \rightarrow\left(\Delta \otimes{ }_{K} P_{0}\right) \otimes{ }_{\Delta}\left(\Delta \otimes{ }_{K} Q_{0}\right)$ is a $\Delta$ - $\Delta$-isomorphism such that the diagram

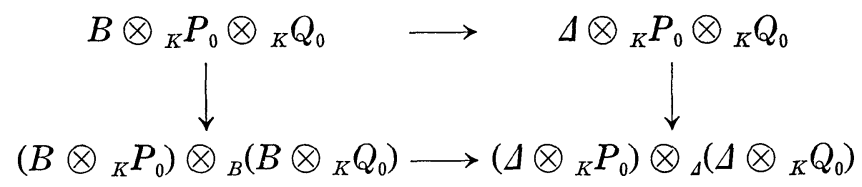

is commutative. Hence $\beta:\left[\phi_{0}\right] \mapsto[\phi]$ is a homomorphism from $P_{K}\left(\oplus K u_{\sigma} / K\right)^{(G)}$ to $P_{K}(\Delta / B)^{(G)}$.

THEOREM 2.15. There is a commutative diagram with exact rows: $U(K) \longrightarrow \operatorname{Aut}\left(\oplus K u_{\sigma} / K\right)^{(G)} \longrightarrow P_{K}\left(\oplus K u_{\sigma} / K\right)^{(G)} \longrightarrow \operatorname{Pic}_{K}(K)^{G}$

(1)

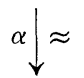

$(K) \longrightarrow \operatorname{Aut}(\Delta / B)^{(G)} \longrightarrow P_{K}(\Delta / B)$

(2)

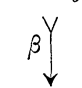

$P_{K}(\Delta / B)^{(G)}$

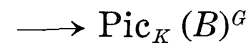

$\longrightarrow C_{0}\left(\oplus K u_{\sigma} / K\right) \longrightarrow B\left(\oplus K u_{\sigma} / K\right) \longrightarrow H^{1}\left(G, \operatorname{Pic}_{K}(K)\right) \longrightarrow H^{3}(G, U(K))$
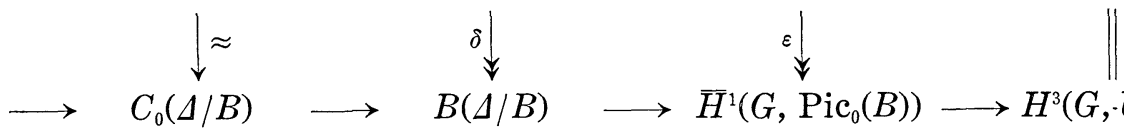

$\bar{H}^{1}\left(G, \operatorname{Pic}_{0}(B)\right) \longrightarrow H^{3}(G, \cdot U(K))$

where $\alpha$ is Aut $\left(\oplus K u_{\sigma} / K\right)^{(G)} \stackrel{\approx}{\longrightarrow} Z^{1}(G, U(K)) \stackrel{\approx}{\longrightarrow} \operatorname{Aut}(\Delta / B)^{(G)}($ cf. Remark to Th. 2.12). and $\beta$ is the homomorphism defined above. 
Proof. By Cor. to Prop. 2.9 and the definition of $\bar{H}^{1}\left(G, \operatorname{Pic}_{0}(B)\right)$, $\varepsilon$ is surjective, and hence so is $\delta$. As $\gamma$ is injective, so is $\beta$, if (1) and (2) are commutative. Therefore it suffices to prove that (1) and (2) are commutative. However the commutativity of (1) is evident. To prove the commutativity of (2), let $\alpha\left(f_{0}\right)=f$. Then, for any $\sigma \in G$, there exists uniquely $c_{\sigma} \in U(K)$ such that $f\left(x_{\sigma}\right)=c_{\sigma} x_{\sigma}$ for all $x_{\sigma} \in J_{\sigma}$. Then $f_{0}\left(u_{\sigma}\right)=$ $c_{\sigma} u_{\sigma}$ for all $\sigma \in G$, and so $\left(x_{\sigma} \otimes u_{f_{0}}\right) x_{\tau}=x_{\sigma} x_{\tau} \otimes u_{\tau-1} u_{f_{0}} u_{\tau}=x_{\sigma} x_{\tau} \otimes u_{\tau-1} c_{\tau} u_{\tau} u_{f_{0}}$ $=x_{\sigma} x_{\tau} \otimes \tau^{-1}\left(c_{\tau}\right) u_{f_{0}}=x_{\sigma} \cdot f\left(x_{\tau}\right) \otimes u_{f_{0}}$ in $\Delta \otimes{ }_{K} K u_{f_{0}}$, where $x_{\sigma} \in J_{\sigma}, x_{\tau} \in J_{\tau}$ (cf. the definition of $\beta$ ). This means that (2) is commutative.

THEOREM 2.16. There exists a commutative diagram

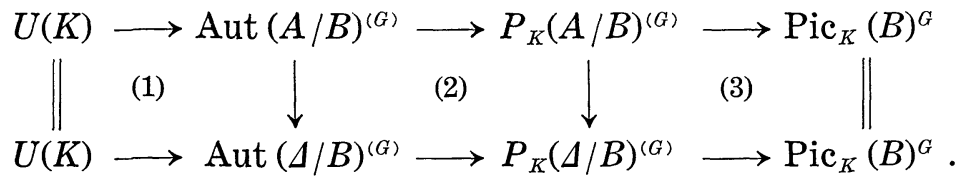

Proof. Let $f$ be in $\operatorname{Aut}(A / B)^{(G)}$. Then $f\left(J_{\sigma}\right)=J_{\sigma}$ for all $\sigma \in G$, so $f$ induces canonically an automorphism of $\Delta / B=\oplus J_{\sigma} / B$. Then the commutativity of (1) is evident. Next we define a homomorphism $P_{K}(A / B)^{(G)}$ $\rightarrow P_{K}(\Delta / B)^{(G)}$. Let $\phi: P \rightarrow M$ be in $P_{K}(A / B)^{(G)}$. For the sake of simplicity, we may assume that $P$ is a submodule of $M$. Then $J_{\sigma} P=J_{\sigma} \otimes$ ${ }_{B} P=P J_{\sigma}=P \otimes{ }_{B} J_{\sigma}$ in $M$ for all $\sigma \in G$. We construct $\oplus J_{\sigma} P$, formally. Then this is isomorphic to $\Delta \otimes_{B} P$ canonically, as $B$ - $B$-modules. Similarly $\oplus P J_{\sigma} \stackrel{\approx}{\longrightarrow} \otimes \otimes_{B} A$. Since $J_{\sigma} P=P J_{\sigma}$, we have an isomorphism $\Delta \otimes{ }_{B} P \stackrel{\approx}{\longrightarrow} P \otimes_{B} \Delta$, as $B$ - $B$-modules. It is easily seen that this isomorphism satisfies the condition of Lemma 1.2. Thus $\bar{\phi}: P \rightarrow \Delta \otimes_{B} P$, $p \mapsto 1 \otimes p$ is in $P_{K}(\Delta / B)^{(G)}$. Let $\psi: Q \rightarrow U$ be another element in $P_{K}(A / B)^{(G)}$. Then $[\phi][\psi]: P \otimes{ }_{B} Q \rightarrow M \otimes{ }_{A} U$. On the other hand, we have $[\bar{\phi}][\bar{\psi}]: P \otimes$ ${ }_{B} Q \rightarrow\left(\Delta \otimes{ }_{B} P\right) \otimes{ }_{\Delta}\left(\Delta \otimes{ }_{B} Q\right)$. Then it is easily seen that the canonical isomorphism $\Delta \otimes_{B} P \otimes_{B} Q \rightarrow\left(\Delta \otimes_{B} P\right) \otimes_{\Delta}\left(\Delta \otimes_{B} Q\right)$ is a $\Delta$ - $\Delta$-isomorphism such that the diagram

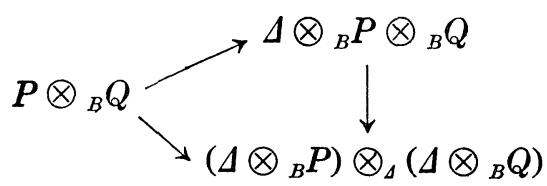

is commutative. Hence the mapping $[\phi] \mapsto[\bar{\phi}]$ is a group homomorphism. Finally, the commutativity of (2) is evident from the definition of the homomorphism $P_{K}(A / B)^{(G)} \rightarrow P_{K}(\Delta / B)^{(G)}$. 
Evidently $1 \rightarrow \operatorname{Aut}\left(A / \Sigma J_{\sigma}\right) \rightarrow \operatorname{Aut}(A / B)^{(G)} \rightarrow \operatorname{Aut}(\Delta / B)^{(G)}$ is exact. Then the commutativity of Th. 2.16 implies that

$$
\operatorname{Aut}\left(A / \Sigma J_{\sigma}\right) \longrightarrow P_{K}(A / B)^{(G)} \longrightarrow P_{K}(\Delta / B)^{(G)}
$$

is exact. Thus we have

COROLlaRY. The following diagram is commutative, and two rows are exact:

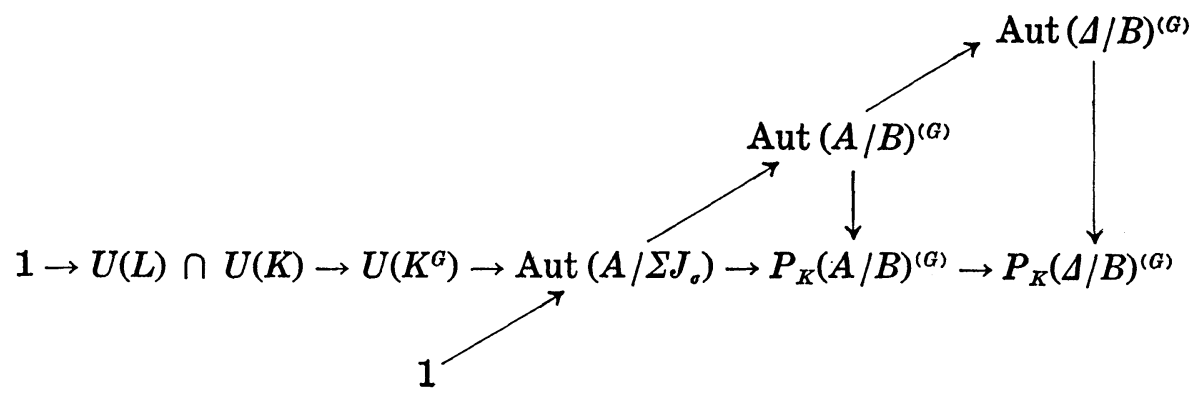

Remark. If $L \subseteq K$ then $\operatorname{Aut}(A / B)^{G}$ is a subgroup of $\operatorname{Aut}(A / B)^{(G)}$. On the other hand, if $V_{\Delta}(B)=K$ then $\operatorname{Aut}(\Delta / B)^{(G)}=\operatorname{Aut}(\Delta / B)$, because $\operatorname{Hom}\left({ }_{B} J_{\sigma B},{ }_{B} J_{\tau B}\right)=0$ for any $\sigma \neq \tau$ (cf. $[17 ; \S 6]$ ).

§3. In this section, $G$ is a group, and $B \supseteq T$ are rings with a common identity. We fix a group homomorphism $G \rightarrow \operatorname{Aut}_{l}(B / T)$ (the group of all $T$-automorphisms of $B / T), \sigma \mapsto \bar{\sigma}$, and we consider $B$ as a $G$-group. $K$ and $F$ are centers of $B$ and $T$, respectively. We put $\Delta_{1}=$ $\oplus_{\sigma \in G} B u_{\sigma} / B$, which is a crossed product of $B$ and $G$ with trivial factor set: $u_{\sigma} u_{\tau}=u_{\sigma \tau}, u_{\sigma} b=\sigma(b) u_{\sigma}$. We denote by $C_{1}$ the center of $\Delta_{1}$. Then, applying Th. 2.12 in $\S 2$ to this generalized crossed product, we obtain an exact sequence

$$
\begin{aligned}
1 \longrightarrow U\left(C_{1}\right) \cap U(K) & \longrightarrow U(K) \longrightarrow \operatorname{Aut}\left(\Delta_{1} / B\right)^{(G)} \longrightarrow P_{K}\left(\Delta_{1} / B\right)^{(G)} \\
& \longrightarrow \operatorname{Pic}_{K}(B)^{G} \longrightarrow C_{0}\left(\Delta_{1} / B\right) \longrightarrow B\left(\Delta_{1} / B\right) \\
& \longrightarrow \bar{H}^{1}\left(G, \operatorname{Pic}_{0}(B)\right) \longrightarrow H^{3}(G, U(K))
\end{aligned}
$$

where $\operatorname{Aut}\left(\Delta_{1} / B\right)^{(G)} \stackrel{\approx}{\longrightarrow} Z^{1}(G, U(K))$ and $C_{0}\left(\Delta_{1} / B\right) \stackrel{\approx}{\longrightarrow} H^{2}(G, U(K))$.

We begin this section with the following

Proposition 3.1. The following two exact sequences consist of G-homomorphisms: 


$$
\begin{aligned}
& 1 \longrightarrow U(K) \cap U(F) \longrightarrow U(K) \longrightarrow \circlearrowleft(B / T) \longrightarrow P(B / T) \longrightarrow \operatorname{Pic}(B), \\
& 1 \longrightarrow U(F) \longrightarrow U\left(V_{B}(T)\right) \longrightarrow \mathbb{S}(B / T) \longrightarrow \operatorname{Pic}(T) .
\end{aligned}
$$

Proof. The exactness was proved in Th. 1.4 and Prop. 1.6. Canonically $\mathbb{S}(B / T)$ is a $G$-group, and the homomorphism $G \rightarrow \operatorname{Aut}(B / T)$ induces a homomorphism $G \rightarrow \operatorname{Aut}(K)$, by restriction. By Th. 1.5, there is a homomorphism Aut $(B / T) \rightarrow P(B / T)$, and this defines a G-group $P(B / T)$, by conjugation. Then it is evident that $P(B / T) \rightarrow \operatorname{Pic}(B)$ is a $G$-homomorphism. Next we shall show that $\mathbb{S}(B / T) \rightarrow P(B / T)$ is a $G$ homomorphism. Let $\sigma \in \operatorname{Aut}(B / T)$, and $X \in \mathbb{S}(B / T)$. Then $\sigma(X) \in \mathbb{S}(B / T)$, and the image of $X$ in $P(B / T)$ is $\phi_{X}: X \rightarrow B, x \mapsto x$. On the other hand the image of $\sigma$ in $P(B / T)$ is $\phi_{\sigma}: T \rightarrow B u_{\sigma}, t \mapsto t u_{\sigma}$. Then there is a commutative diagram

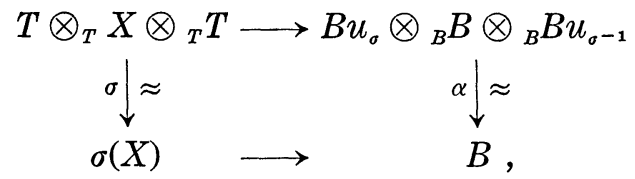

where $\alpha$ is the canonical one. This shows that $\mathbb{S}(B / T) \rightarrow P(B / T)$ is a $G$-homomorphism. It is easily seen that $U\left(V_{B}(T)\right) \rightarrow \mathbb{S}(B / T), d \mapsto T d$ is a $G$-homomorphism.

We denote by $\mathscr{S}(B / T)^{(G)}$ the group $\{X \in \mathbb{S}(B / T) \mid X(\bar{\sigma})=\bar{\sigma}$ for all $\sigma \in G\}$, where $\tilde{\sigma}$ denotes the image of $\sigma$ in Aut $(B / T)$ (cf. Prop. 1.1). In $\S 1$, we have seen that $\mathbb{S}(B / T)^{(G)}=\{X \in \mathbb{S}(B / T) \mid u(X, \bar{\sigma}, 1) \in K\}=\{X \in \mathbb{S}(B / T) \mid$ for any $\sigma \in G$, there exists $c_{\sigma} \in U(K)$ such that $c_{\sigma} x=\sigma(x)$ for all $\left.x \in X\right\}$. We denote by $P^{K}(B / T)^{(G)}$ the subgroup of $P^{K}(B / T)$ (cf. $\S 1$ ), which consists of all $[\phi]$ satisfying $(* *)$.

(**) For any $\sigma \in G$, there exists a $B$ - $B$-isomorphism $f_{\sigma}: M \rightarrow B u_{\sigma}$ $\otimes_{B} M \otimes_{B} B u_{\sigma-1}$ such that the diagram

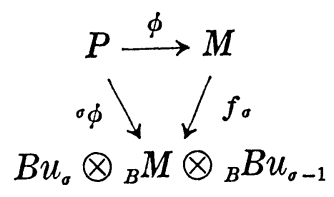

is commutative, where ${ }^{\circ} \phi$ is the map $p \mapsto u_{\sigma} \otimes \phi(p) \otimes u_{\sigma-1}(p \in P)$. The proof that $P^{K}(B / T)^{(G)}$ is a subgroup is the following

Proposition 3.2. $P^{K}(B / T)^{(G)}$ is a subgroup of $P^{K}(B / T)$. 
Proof. Let $\phi: P \rightarrow M$ and $\psi: Q \rightarrow U$ be two representations of an element of $P^{K}(B / T)^{(G)}$, and let the diagram

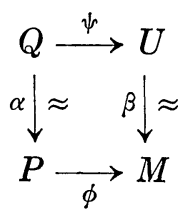

be commutative, where $\alpha$ is a $T$-T-isomorphism, and $\beta$ is a $B-B$ isomorphism. For any $\sigma$ in $G$, there is a $B$-B-isomorphism $f_{\sigma}: M \rightarrow$ $B u_{\sigma} \otimes_{B} M \otimes_{B} B u_{\sigma-1}$ such that the diagram

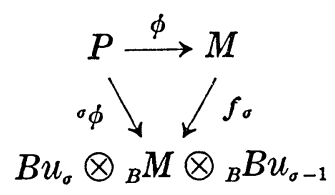

is commutative. Then a $B$-B-isomorphism $g_{\sigma}: U \rightarrow B u_{\sigma} \otimes{ }_{B} U \otimes_{B} B u_{\sigma-1}$ is determined by the commutativity of the following diagram:

$$
\begin{aligned}
& Q \stackrel{\psi}{\longrightarrow} U \stackrel{g_{\sigma}}{\longrightarrow} B u_{\sigma} \otimes{ }_{B} U \otimes{ }_{B} B u_{\sigma-1}, \\
& \alpha \downarrow \approx \quad \beta|\approx \quad 1 \otimes \beta \otimes 1| \approx \\
& P \underset{\phi}{\longrightarrow} M \underset{f_{\sigma}}{\longrightarrow} B u_{\sigma} \otimes{ }_{B} M \otimes{ }_{B} B u_{\sigma-1}
\end{aligned}
$$

that is, $g_{\sigma}=(1 \otimes \beta \otimes 1)^{-1} f_{\sigma} \beta$. It is easily seen that $g_{\sigma} \psi(q)=u_{\sigma} \otimes \psi(q)$ $\otimes u_{\sigma-1}(q \in Q)$, and hence $P^{K}(B / T)^{(G)}$ is well defined. It is evident that $P^{K}(B / T)^{(G)}$ is closed under multiplication. Finally $f_{\sigma}:{ }_{B} M_{B} \rightarrow{ }_{B} B u_{\sigma} \otimes{ }_{B} M$ $\otimes{ }_{B} B u_{\sigma-1 B}$ induces a $B$-B-isomorphism $\operatorname{Hom}_{r}\left({ }_{B} M,{ }_{B} B\right) \stackrel{\approx}{\longrightarrow} \operatorname{Hom}_{r}\left({ }_{B} B u_{\sigma} \otimes\right.$ $\left.{ }_{B} M \otimes{ }_{B} B u_{\sigma-1},{ }_{B} B\right)$, and there is a canonical $B$-B-isomorphism $B u_{\sigma} \otimes$ ${ }_{B} \mathrm{Hom}_{r}\left({ }_{B} M,{ }_{B} B\right) \otimes{ }_{B} B u_{\sigma-1} \rightarrow \operatorname{Hom}_{r}\left({ }_{B} B u_{\sigma} \otimes{ }_{B} M \otimes{ }_{B} B u_{\sigma-1},{ }_{B} B\right), u_{\sigma} \otimes h \otimes u_{\sigma-1} \mapsto$ $\left(u_{\sigma} \otimes x \otimes u_{\sigma-1} \rightarrow \sigma\left(x^{h}\right)\right)(x \in M)$. Then we have a commutative diagram:

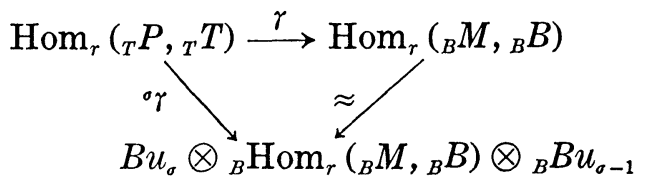

where $\gamma$ is the canonical homomorphism $f \mapsto\left(\phi(p) \rightarrow p^{f}\right)(p \in P)$. This completes the proof. 
THEOREM 3.3. There is an exact sequence

$$
U(K) \longrightarrow \mathbb{S}(B / T)^{(G)} \longrightarrow P^{K}(B / T)^{(G)} \longrightarrow \operatorname{Pic}_{K}(B)^{G}
$$

Proof. For $X$ in $\circlearrowleft(B / T)$, the image of $X$ in $\operatorname{Pic}^{K}(B / T)$ is the canonical inclusion map $\phi: X \rightarrow B$. Then ${ }^{\circ} \phi$ is $X \rightarrow B, x \mapsto \sigma(x)$. Therefore $[\phi]$ is in $\operatorname{Pic}^{K}(B / T)^{(G)}$ if and only if, for any $\sigma \in G$, there is a $c_{\sigma} \in U(K)$ such that $c_{\sigma} x=\sigma(x)$ for all $x \in X$, that is, $X \in \mathbb{S}(B / T)^{(G)}$. Then the exactness of the present sequence follows from Th. 1.4.

THEOREM 3.4. There is a commutative diagram with exact rows:

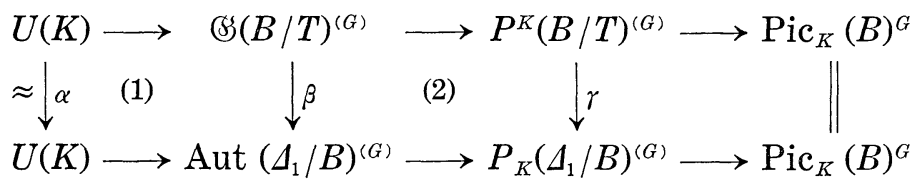

Proof. The isomorphism $U(K) \stackrel{\alpha}{\longrightarrow} U(K)$ is $c \mapsto c^{-1}$. Let $X \in \mathbb{B S}(B / T)^{(G)}$. Then, for any $\sigma$ in $G$, there exists uniquely $c_{\sigma} \in U(K)$ such that $c_{\sigma} x=\sigma(x)$ for all $x \in X$. If is easily seen that $c_{\sigma \tau}=c_{\sigma} \cdot \sigma\left(c_{\tau}\right)$ for all $\sigma, \tau \in G, c_{1}=1$. Then $c_{\sigma}(\sigma \in G)$ defines an automorphism $\rho: \sum_{\sigma} b_{\sigma} u_{\sigma} \mapsto \sum_{\sigma} b_{\sigma} c_{\sigma} u_{\sigma}$. We define $\mathbb{S}(B / T)^{(G)} \stackrel{\beta}{\longrightarrow}$ Aut $\left(\Delta_{1} / B\right)^{(G)}, X \mapsto \rho . \quad$ The commutativity of (1) is easily seen. Next we shall define $P^{K}(B / T)^{(G)} \stackrel{r}{\longrightarrow} P_{K}\left(\Delta_{1} / B\right)^{(G)}$. Let $\phi: P \rightarrow M$ be in $P^{K}(B / T)^{(G)}$. Then, for any $\sigma \in G$, there exists a $B$-B-isomorphism $f_{\sigma}: M \rightarrow B u_{\sigma} \otimes{ }_{B} M \otimes{ }_{B} B u_{\sigma-1}$ such that $f_{\sigma} \phi={ }^{\sigma} \phi$. Then $f_{\sigma}$ induces an isomorphism $f_{\sigma}^{\prime}: M \otimes{ }_{B} B u_{\sigma} \stackrel{f_{\sigma} \otimes 1}{\longrightarrow} B u_{\sigma} \otimes{ }_{B} M \otimes{ }_{B} B u_{\sigma-1} \otimes_{B} B u_{\sigma} \stackrel{*}{\longrightarrow} B u_{\sigma} \otimes_{B} M$, where $*$ is induced by the canonical map $B u_{\sigma_{-1}} \otimes_{B} B u_{\sigma} \rightarrow B$. As is easily seen, $f_{\sigma}^{\prime}\left(\phi(p) \otimes u_{o}\right)=u_{\sigma} \otimes \phi(p)(p \in P)$. Taking direct sum, we have an isomorphism $\Delta_{1} \otimes{ }_{B} M \stackrel{\approx}{\longrightarrow} M \otimes_{B} \Delta_{1}$, and it is easy to check that this isomorphism satisfies the condition of Lemma 1.2. Thus we have $\bar{\phi}: M \rightarrow$ $\Delta_{1} \otimes{ }_{B} M, m \mapsto 1 \otimes m$, in $P_{K}\left(\Delta_{1} / B\right)^{(G)}$ (cf. $\S 2$ ). Let $\psi: Q \rightarrow U$ be another element in $P^{K}(B / T)^{(G)}$. Then the canonical isomorphism $\Delta_{1} \otimes{ }_{B} M \otimes_{B} U \stackrel{\approx}{\longrightarrow}$ $\left(\Delta_{1} \otimes{ }_{B} M\right) \otimes_{A_{1}}\left(\Delta_{1} \otimes{ }_{B} U\right)$ is a $\Delta_{1}-\Delta_{1}$-isomorphism such that the diagram

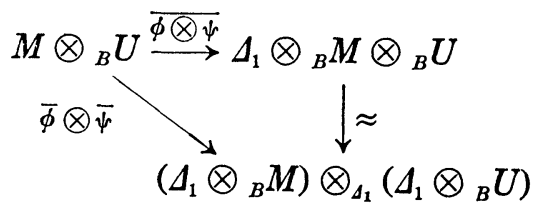

is commutative. Hence the map $\phi \rightarrow \bar{\phi}$ is a homomorphism. Finally we shall show the commutativity of (2). Let $1=\sum_{i} x_{i}^{\prime} x_{i}\left(x_{i}^{\prime} \in X^{-1}, x_{i} \in X\right)$. 
Then $\Delta_{1} \otimes{ }_{B} B \ni u_{\sigma} \otimes 1=\sum_{i} u_{\sigma} x_{i}^{\prime} \otimes x_{i}, \quad$ so $\quad\left(u_{\sigma} \otimes 1\right) u_{\tau}=\left(\sum_{i} u_{\sigma} x_{i}^{\prime} \otimes x_{i}\right) u_{\tau}=$ $\left(\sum_{i} \sigma\left(x_{i}^{\prime}\right) u_{\sigma} \otimes x_{i}\right) u_{\tau}=\sum_{i} \sigma\left(x_{i}^{\prime}\right) u_{\sigma} u_{\tau} \otimes x_{i}=\sum_{i} u_{\sigma} x_{i}^{\prime} u_{\tau} \otimes x_{i}=\sum_{i} u_{\sigma} x_{i}^{\prime} u_{\tau} x_{i} \otimes 1=$ $\sum_{i} u_{\sigma} x_{i}^{\prime} x_{i} c_{\tau} u_{\tau} \otimes 1=u_{\sigma} \cdot \rho\left(u_{\tau}\right) \otimes 1$. Hence $\Delta_{1} \otimes{ }_{B} B \stackrel{\approx}{\longrightarrow} \Delta_{1} u_{\rho}, u_{\sigma} \otimes 1 \mapsto u_{\sigma} u_{\rho}$ is a $\Delta_{1}-\Delta_{1}$-isomorphism. Hence (2) is commutative. This completes the proof.

The next Cor. 1 is follows from Th. 3.4.

COROLlaRY 1. The following diagram is commutative, and two rows are exact:

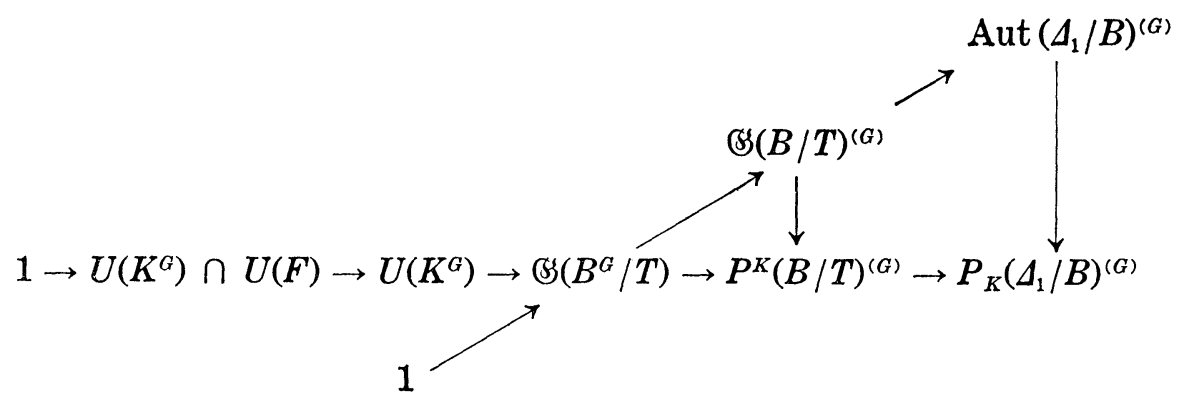

where $K$ and $F$ are centers of $B$ and $T$, respectively.

Corollary 2. If $B^{G}=T$ then two homomorphisms $\quad \&(B / T)^{(G)} \rightarrow$ Aut $\left(\Delta_{1} / B\right)^{(G)}$ and $P^{K}(B / T)^{(G)} \rightarrow P_{K}\left(\Delta_{1} / B\right)^{(G)}$ are monomorphisms. Therefore, in this case, $\mathbb{S}(B / T)^{(G)}$ is an abelian group.

COROLlary 3. If $B / T$ is a finite $G$-Galois extension, then all vertical maps in Th. 3.4 are isomorphisms.

Proof. It suffices to prove that $\gamma$ is surjective, by Cor. 2, Th. 1.4. and Th. 1.5, because the center of $\Delta_{1}$ is $F$ in this case. Let $\bar{\phi}: M \rightarrow \bar{M}$ be in $P_{K}\left(\Delta_{1} / B\right)^{(G)}$, and let $M \subseteq \bar{M}$. Then, $u_{\sigma} M=M u_{\sigma}(\sigma \in G)$, and this yields a left $\Delta_{1}$-module $M: u_{\sigma} * m=u_{\sigma} m u_{\sigma-1}(m \in M, \sigma \in G)$. Then, by [8; Th. 1.3], $M=B \otimes_{T} M_{0}$, where $M_{0}=\left\{m \in M \mid u_{\sigma} m=m u_{\sigma}\right.$ for all $\left.\sigma=G\right\}$. Similarly $M=M_{0} \otimes_{T} B$, and the inclusion map $\phi: M_{0} \rightarrow M$ is in $P^{K}(B / T)^{(G)}$, because ${ }_{T} M_{0 T} \stackrel{\approx}{\longrightarrow} \operatorname{Hom}_{r}\left({ }_{A_{1}} B,{ }_{\Delta_{1}} M\right)_{T}$ is a Morita module. By the proof of Th. 3.4, $\gamma(\phi)=\bar{\phi}$ is easily seen.

Proposition 3.5. If $V_{B}(T)=K$ then $\operatorname{ss}(B / T)^{(G)}=(B)(B / T)$.

Proof. Let $X \in \mathbb{S}(B / T)$, and let $1=\sum_{i} a_{i} a_{i}^{\prime}\left(a_{i} \in X, a_{i}^{\prime} \in X^{-1}\right)$, and $\sigma \in G$. Then $u=\sum_{i} a_{i} \cdot \sigma\left(a_{i}^{\prime}\right) \in V_{B}(T)=K$, and $u \cdot \sigma(x)=x$ for all $x \in X$ (cf. $\S 1$ ). 


\section{§4. Morita invariance of the exact sequence in $\S 2$.}

In this section we shall cast a glance at the Morita invariance of the exact sequence in Th. 2.12. We fix two Morita modules ${ }_{A} M_{A^{\prime}} \supseteq{ }_{B} P_{B^{\prime}}$ such that $M=A \otimes{ }_{B} P=P \otimes_{B^{\prime}} A^{\prime}$ (cf, [19]), where $B \subseteq A$ and $B^{\prime} \subseteq A^{\prime}$. We put $V_{A}(A)=L, V_{A^{\prime}}\left(A^{\prime}\right)=L^{\prime}, V_{B}(B)=K$, and $V_{B^{\prime}}\left(B^{\prime}\right)=K^{\prime}$. There is an isomorphism $V_{A}(B) \rightarrow V_{A^{\prime}}\left(B^{\prime}\right), c \mapsto c^{\prime}$ such that $c p=p c^{\prime}$ for all $p \in P$, and this induces $L \stackrel{\approx}{\longrightarrow} L^{\prime}$ and $K \stackrel{\approx}{\longrightarrow} K^{\prime}$, by [19; Prop. 3.3]. Further, by [19; Th. 3.5], Aut $(A / B) \stackrel{\approx}{A} \operatorname{Aut}\left(A^{\prime} / B^{\prime}\right), \sigma \mapsto \sigma^{\prime}$, where $\sum \sigma\left(a_{i}\right) p_{i}=$ $\sum q_{j} \cdot \sigma^{\prime}\left(a_{j}^{\prime}\right)$ for all $\sum a_{i} p_{i}=\sum q_{j} a_{j}^{\prime}\left(a_{i} \in A, p_{i}, q_{j} \in P, a_{j}^{\prime} \in A^{\prime}\right)$ in $M$. Then it is evident the diagram

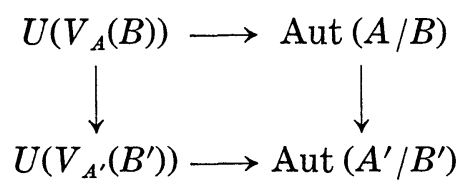

is commutative. Let $\sigma \mapsto \sigma^{\prime}$ under the isomorphism Aut $(A / B) \rightarrow$ Aut $\left(A^{\prime} / B^{\prime}\right)$. Then $A u_{\sigma} \otimes{ }_{A} M \rightarrow M \otimes{ }_{A^{\prime}} A^{\prime} u_{\sigma^{\prime}}, u_{\sigma} \otimes p \mapsto p \otimes u_{\sigma^{\prime}}(p \in P)$ is an $A$ - $A^{\prime}$-isomorphism. Hence

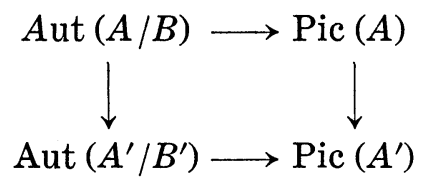

is a commutative diagram, where $\operatorname{Pic}(A) \rightarrow \operatorname{Pic}\left(A^{\prime}\right),[X] \mapsto\left[X^{\prime}\right]$ is the isomorphism such that $X \otimes{ }_{A} M \stackrel{\approx}{\longrightarrow} M \otimes{ }_{A^{\prime}} X^{\prime}$ as $A$ - $A^{\prime}$-modules. There is an isomorphism $\mathbb{S}(A / B) \rightarrow \circlearrowleft\left(A^{\prime} / B^{\prime}\right), Y \mapsto Y^{\prime}$ such that $Y P=P Y^{\prime}$ (cf. [19; Prop. 3.3]). Then the following diagram is commutative:

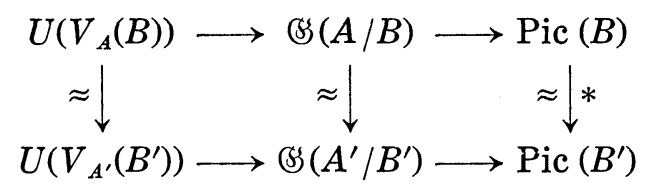

where $*:[W] \mapsto\left[W^{\prime}\right]$ is the isomorphism such that $W \otimes{ }_{B} P \stackrel{\approx}{\longrightarrow} P \otimes_{B^{\prime}} W^{\prime}$ as $B$ - $B^{\prime}$-modules. The isomorphism $P(A / B) \rightarrow P\left(A^{\prime} / B^{\prime}\right), \phi: Q \rightarrow U \mapsto$ $\phi^{\prime}: Q^{\prime} \rightarrow U^{\prime}$ is defined by the commutativity of the diagram

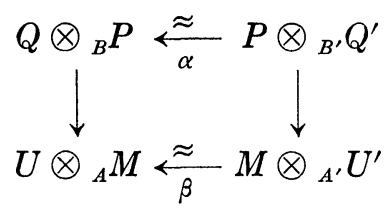


for some $B$ - $B^{\prime}$-isomorphism $\alpha$ and some $A$-A'-isomorphism $\beta$. In fact, we put $Q^{\prime}=\operatorname{Hom}_{r}\left({ }_{B} P,{ }_{B} B\right) \otimes{ }_{B} Q \otimes{ }_{B} P$ and $U^{\prime}=\operatorname{Hom}_{r}\left({ }_{A} M,{ }_{A} A\right) \otimes{ }_{A} U \otimes{ }_{A} M$, and take the canonical isomorphisms $P \otimes \otimes_{B^{\prime}} Q^{\prime} \stackrel{\approx}{\longrightarrow} Q \otimes_{B} P$ and $M \otimes_{A^{\prime}} U^{\prime}$ $\stackrel{\approx}{\longrightarrow} U \otimes_{A} M$. Then it is clear that the following diagrams are commutative:

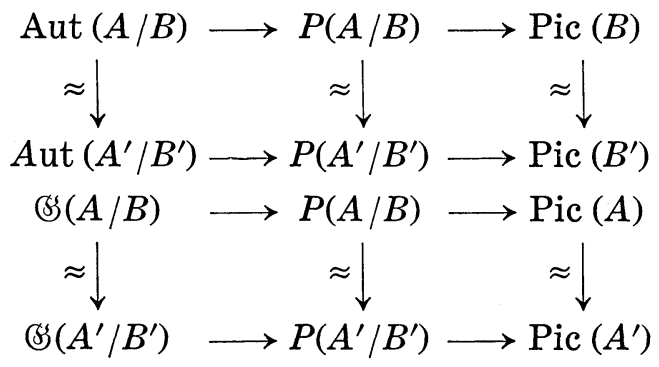

We now fix a commutative diagram

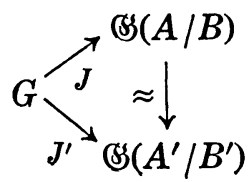

consisting of group homomorphisms. Put $\Delta=\oplus J_{\sigma} / B$ and $\Delta^{\prime}=\oplus J_{\sigma}^{\prime} / B^{\prime}$. Then we have

THEOREM 4.1. There exists a commutative diagram

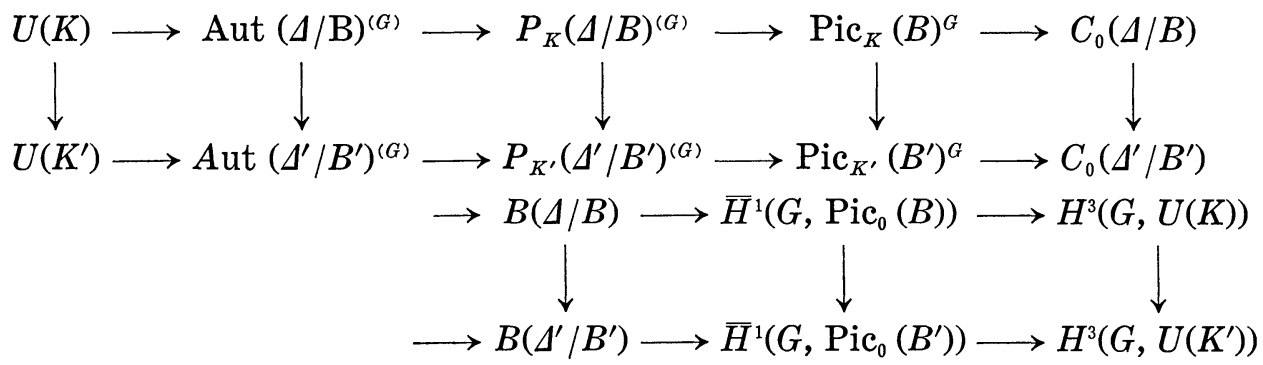

where all vertical maps are isomorphisms.

Proof. First we shall show that there is an isomorphism $C(\Delta / B)$ $\stackrel{\approx}{\longrightarrow} C\left(\Delta^{\prime} / B^{\prime}\right), \oplus U_{\sigma} / B \mapsto \oplus U_{\sigma}^{\prime} / B^{\prime}$. Put $P^{*}=\operatorname{Hom}_{r}\left({ }_{B} P,{ }_{B} B\right)$ and $P^{*} \otimes{ }_{B} U_{\sigma}$ $\otimes P=U_{\sigma}^{\prime}$. Then, for any $\sigma \in G$, there is a canonical $B$ - $B^{\prime}$-isomorphism $f_{\sigma}: U_{\sigma} \otimes{ }_{B} P \rightarrow P \otimes{ }_{B^{\prime}} P^{*} \otimes_{B} U_{\sigma} \otimes{ }_{B} P=P \otimes{ }_{B^{\prime}} U_{\sigma}^{\prime}$. The multiplicalion in $\oplus U_{\sigma}^{\prime} / B$ is defined by the commutativity of the diagram 


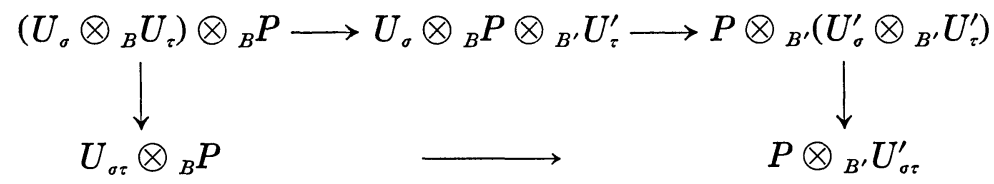

The isomorphism $\oplus f_{\sigma}:\left(\oplus U_{\sigma}\right) \otimes_{B} P \rightarrow P \otimes_{B^{\prime}}\left(\oplus U_{\sigma}^{\prime}\right)$ satisfies the condition in Lemma 1.2, and $f_{\sigma}$ induces an isomorphism $U_{\sigma} \otimes{ }_{B} P \rightarrow P \otimes{ }_{B^{\prime}} U_{\sigma}^{\prime}$, that is, $\oplus U_{\sigma} / B$ and $\oplus U_{\sigma}^{\prime} / B^{\prime}$ defined above are equivalent as generalized crossed products. In particular, $\Delta / B$ and $\Delta^{\prime} / B^{\prime}$ are equivalent. The isomorphism $\mathrm{Pic}(B) \rightarrow \operatorname{Pic}\left(B^{\prime}\right)$ induces the isomorphism $\operatorname{Pic}_{K}(B)^{[G]} \rightarrow$ $\operatorname{Pic}_{K^{\prime}}\left(B^{\prime}\right)^{[G]},[W] \mapsto\left[P^{*} \otimes_{B} W \otimes_{B} P\right]$, where $P^{*}=\operatorname{Hom}_{r}\left({ }_{B} P,{ }_{B} B\right)$. We put $W^{\prime}=P^{*} \otimes{ }_{B} W \otimes_{B} P$. Then $W^{* \prime} \approx W^{*}$ canonically, where $W^{*}=$ $\operatorname{Hom}_{r}\left({ }_{B^{\prime}} W^{\prime},{ }_{B^{\prime}} B^{\prime}\right)$. Noting this fact, we can see that the diagram

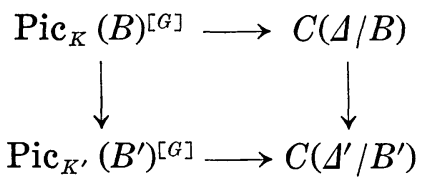

is commutative. The isomorphism $\mathrm{Pic}_{0}(B) \rightarrow \mathrm{Pic}_{0}\left(B^{\prime}\right)$ induces the isomorphism $Z^{1}\left(G, \operatorname{Pic}_{0}(B)\right) \rightarrow Z^{1}\left(G, \operatorname{Pic}_{0}\left(B^{\prime}\right)\right.$ ) (cf. Cor. to Prop. 2.9), and it is evident the diagram

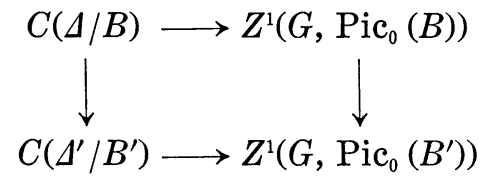

is commutative. The facts that the isomorphism $P(\Delta / B) \rightarrow P\left(\Delta^{\prime} / B^{\prime}\right)$ induces $P_{K}(\Delta / B)^{(G)} \stackrel{\approx}{\longrightarrow} P_{K^{\prime}}\left(\Delta^{\prime} / B^{\prime}\right)^{(G)}$, and that the isomorphism Aut $(\Delta / B)$ $\rightarrow$ Aut $\left(\Delta^{\prime} / B^{\prime}\right)$ induces Aut $(\Delta / B)^{(G)} \stackrel{\approx}{\approx}$ Aut $\left(\Delta^{\prime} / B^{\prime}\right)^{(G)}$ are easily checked. After these remarks it is easy to complete the proof.

If we take a commutative diagram

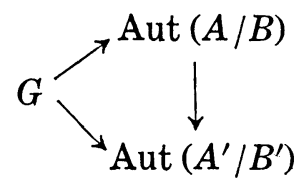

then each $g_{\sigma}: A u_{\sigma} \otimes{ }_{A} M \rightarrow M \otimes{ }_{A^{\prime}} A^{\prime} u_{\sigma}^{\prime}, u_{\sigma} \otimes p \mapsto p \otimes u_{\sigma}^{\prime}(p \in P)$ is an $A-A^{\prime}-$ isomorphism, and $\oplus g_{\sigma}:\left(\oplus A u_{\sigma}\right) \otimes_{A} M \rightarrow M \otimes A_{A^{\prime}}\left(\oplus A^{\prime} u_{\sigma}^{\prime}\right)$ satisfies the condition of Lemma 1.2 , so that $\oplus A u_{\sigma} / B$ and $\oplus A^{\prime} u_{\sigma}^{\prime} / B^{\prime}$ with trivial factor 
set are equivalent as generalized crossed products. Therefore Th. 4.1 is applicable to this case.

§5. In this section we fix a Morita module ${ }_{A / B} M_{B^{*} / A^{*}}$ (cf. [19]) and a commutative diagram

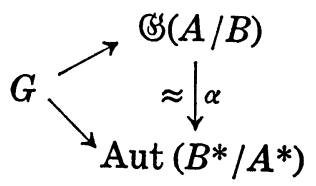

of group homomorphisms, where $\alpha: X \mapsto \sigma$ is defined by $(x m) \cdot \sigma\left(b^{*}\right)=$ $x\left(m b^{*}\right)\left(x \in X, m \in M, b^{*} \in B^{*}\right)$ (cf. [19; Th. 1.5]), and $A \supseteq B$ and $B^{*} \supseteq A^{*}$ are rings. For any $c$ in $V_{A}(B)$, there is a $c^{\prime} \in V_{B^{*}}\left(A^{*}\right)$ such that $c m=$ $m c^{\prime}$ for all $m \in M$. Then the map $c \mapsto c^{\prime-1}$ is a group isomorphism $U\left(V_{A}(B)\right) \rightarrow U\left(V_{B^{*}}\left(A^{*}\right)\right)$, and this induces isomorphisms $U(K) \rightarrow U\left(K^{*}\right)$, $U(L) \rightarrow U\left(L^{*}\right)$, where $K=V_{B}(B), K^{*}=V_{B^{*}}\left(B^{*}\right), L=V_{A}(A)$, and $L^{*}=$ $V_{A^{*}}\left(A^{*}\right)$. The following diagram is commutative:

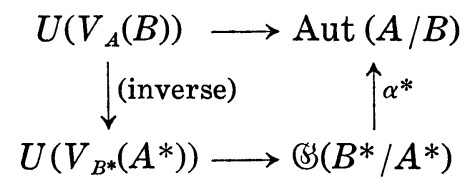

where $\alpha^{*}: X^{*} \mapsto \sigma^{*}$ is defined by $\left(\sigma^{*}(a) m\right) x^{*}=a\left(m x^{*}\right)\left(x^{*} \in X^{*}, m \in M\right.$, $a \in A)$, or equivalently, $\sigma^{*}(a)\left(m y^{*}\right)=(a m) y^{*}\left(y^{*} \in X^{*-1}\right)$.

Proposition 5.1. Aut $(A / B)^{(G)} \underset{\mathbb{S}}{\longrightarrow}\left(B^{*} / A^{*}\right)^{(G)}$.

Proof. Let $X \mapsto \sigma$ under the isomorphism $\mathbb{S}(A / B) \rightarrow \operatorname{Aut}\left(B^{*} / A^{*}\right)$, and let $\sigma^{*} \mapsto X^{*}$ under the isomorphism Aut $(A / B) \rightarrow \circlearrowleft\left(B^{*} / A^{*}\right)$. Then it suffices to prove that $X\left(\sigma^{*}\right) \mapsto \sigma\left(X^{*}\right)$ under Aut $(A / B) \rightarrow \mathscr{S}\left(B^{*} / A^{*}\right)$. Let $\tau \leftrightarrow \sigma\left(X^{*}\right)$ under Aut $(A / B) \rightarrow \circlearrowleft\left(B^{*} / A^{*}\right)$. There is a $u \in U\left(V_{A}(B)\right)$ such that $X\left(\sigma^{*}\right)(a)=u \cdot \sigma^{*}(a) u^{-1}(a \in A)$ (cf. $\left.\S 1\right)$. Then $u \cdot \sigma^{*}(x)=x$ for all $x \in X$, and so $u \cdot \sigma^{*}(x) m=x m$ for all $m \in M$. Let $y^{*} \in X^{*-1}$. Then $(x m) \cdot \sigma\left(y^{*}\right)$ $=x\left(m y^{*}\right)=u \cdot \sigma^{*}(x)\left(m y^{*}\right)=u\left((x m) y^{*}\right)=(x m) y^{*} u^{\prime}$, so that $\sigma\left(y^{*}\right)=y^{*} u^{\prime}$ for all $y^{*} \in X^{*-1}$, where $u m=m u^{\prime}$ for all $m \in M$. Then, for any $a \in A$, $\tau(a)\left(m \cdot \sigma\left(y^{*}\right)\right)=(a m) \cdot \sigma\left(y^{*}\right)=(a m) y^{*} u^{\prime}=u\left((a m) y^{*}\right)=u \cdot \sigma^{*}(a)\left(m y^{*}\right)=$ $u \cdot \sigma^{*}(a) u^{-1} \cdot u\left(m y^{*}\right)$. But $u\left(m y^{*}\right)=m y^{*} u^{\prime}=m \cdot \sigma\left(y^{*}\right)$. Hence. $\tau(a)=$ $X\left(\sigma^{*}\right)(a)$ for all $a \in A$.

Proposition 5.2. There is an isomorphism $P(A / B) \stackrel{\approx}{\longrightarrow} P\left(B^{*} / A^{*}\right)$. 
Proof. Let $\phi: P \rightarrow N$ be in $P(A / B)$. Put ${ }_{B^{*}} P_{B^{*}}^{\prime}=\operatorname{Hom}_{r}\left({ }_{B} M,{ }_{B} B\right) \otimes$ ${ }_{B} P \otimes{ }_{B} M$ and ${ }_{A^{*}} N_{A^{*}}=\operatorname{Hom}_{r}\left({ }_{A} M,{ }_{A} A\right) \otimes{ }_{A} N \otimes{ }_{A} M$. Then there are canonical isomorphisms ${ }_{B} M \otimes{ }_{B^{*}} P_{B^{*}}^{\prime} \rightarrow{ }_{B} P \otimes{ }_{B} M_{B^{*}}$ and ${ }_{A} M \otimes{ }_{A^{*}} N^{\prime}{ }_{A^{*}} \rightarrow{ }_{A} N \otimes{ }_{A} M_{A^{*}}$. Then $\phi^{\prime}: N^{\prime} \rightarrow P^{\prime}$ in $P_{K^{*}}\left(B^{*} / A^{*}\right)$ is defined by the commutativity of

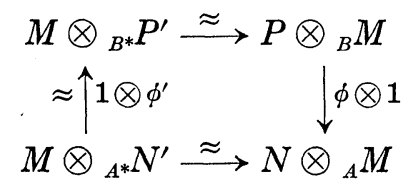

Let $\psi: Q \rightarrow U$ be another element in $P(A / B)$, and $\psi^{\prime}: U^{\prime} \rightarrow Q^{\prime}$ is the one defined by $\psi$. Then the following diagram is commutative:

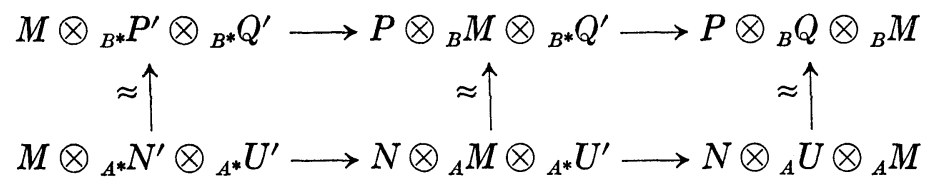

On the other hand we have a diagram

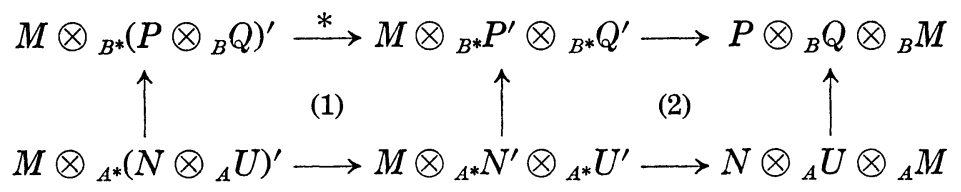

where (2) and (1) $+(2)$ are commutative, and $*$ is induced by $\left(P \otimes_{B} Q\right)^{\prime}$ $\stackrel{\approx}{\longrightarrow} P^{\prime} \otimes{ }_{B^{*}} Q^{\prime}$. Hence (1) is commutative, and this proves that the map $[\phi] \mapsto\left[\phi^{\prime}\right]$ is a homomorphism. Similarly we can define a homomorphism $P\left(B^{*} / A^{*}\right) \rightarrow P(A / B)$. Hence $P(A / B) \stackrel{\approx}{\longrightarrow} P\left(B^{*} / A^{*}\right),[\phi] \mapsto\left[\phi^{\prime}\right]$.

THEOREM 5.3. $\oplus J_{\sigma} / B$ and $\oplus B^{*} u_{\sigma} / B^{*}$ are equivalent by ${ }_{B} M_{B^{*}}$, as generalized crossed products. Therefore Th. 4.1 is applicable to this case.

Proof. For any $\sigma$ in $G$, the map $J_{\sigma} \otimes{ }_{B} M \rightarrow M \otimes{ }_{B^{*}} B^{*} u_{\sigma}, x \otimes m \mapsto$ $x m \otimes u_{\sigma}$ is a $B-B^{*}$-isomorphism, and the following diagram is commutative:

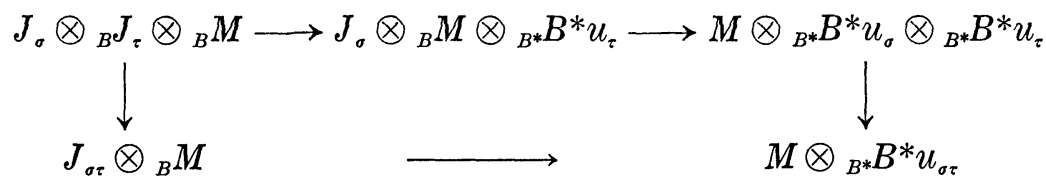

THEOREM 5.4. There is a commutative diagram 


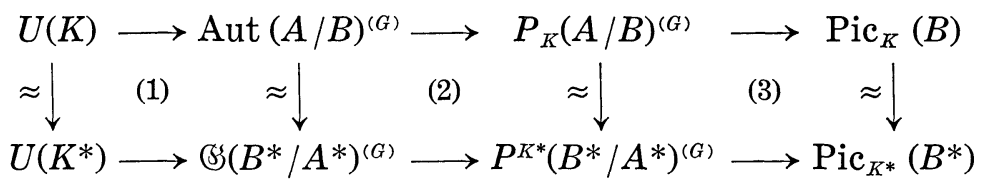

Proof. It suffices to prove that $P(A / B) \stackrel{\approx}{\longrightarrow} P\left(B^{*} / A^{*}\right)$ induces $P_{K}(A / B)^{(G)} \stackrel{\approx}{\longrightarrow} P^{K^{*}}\left(B^{*} / A^{*}\right)^{(G)}$, and that (1), (2), (3) are commutative. Now, $J_{\sigma} \otimes{ }_{B} M \stackrel{\approx}{\longrightarrow} M \otimes{ }_{B *} B^{*} u_{\sigma}, x \otimes m \mapsto x m \otimes u_{\sigma}$, as $B$ - $B^{*}$-modules. Let $\phi: P \rightarrow N$ be in $P_{K}(A / B)^{(G)}$. Then, for any $\sigma$ in $G$, there exists an isomorphism $f_{\sigma}:{ }_{B} J_{\sigma} \otimes{ }_{B} P \otimes{ }_{B} J_{\sigma-1 B} \rightarrow{ }_{B} P_{B}$ such that

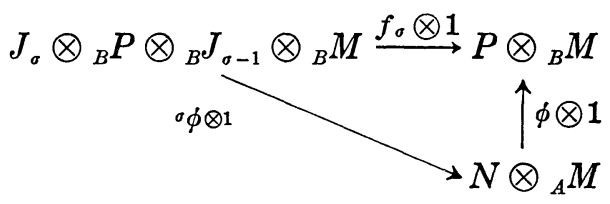

is commutative. Then a $B^{*}-B^{*}$-isomorphism $f_{\sigma}^{\prime}: P^{\prime} \rightarrow B^{*} u_{\sigma} \otimes{ }_{B^{*}} P^{\prime} \otimes$ ${ }_{B^{*}} B^{*} u_{a_{-1}}$ is defined by the commutativity of

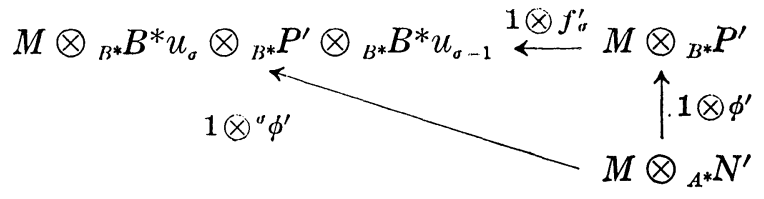

Thus $\left[\phi^{\prime}\right]$ is in $P^{K^{*}}\left(B^{*} / A^{*}\right)^{(G)}$, and hence $P_{K}(A / B)^{(G)} \stackrel{\approx}{\longrightarrow} P^{K^{*}}\left(B^{*} / A^{*}\right)^{(G)}$. The commutativity of (1) and (3) is easily seen. To prove the commutativity of (2), let $\sigma \in \operatorname{Aut}(A / B)^{(G)}$, and $\sigma \mapsto X$ under the isomorphism Aut $(A / B)^{(G)} \rightarrow \mathfrak{S}\left(B^{*} / A^{*}\right)^{(G)}$. Then $M X=M \otimes_{A^{*}} X \stackrel{\approx}{\longrightarrow} A u_{o} \otimes{ }_{A} M, m \otimes x$ $\mapsto u_{\sigma} \otimes m x$ is an $A$ - $A^{*}$-isomorphism. And it is easy to see that the diagram

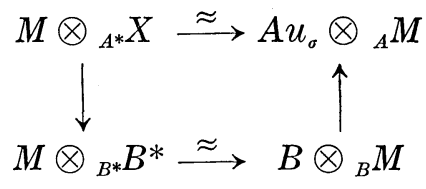

is commutative. Hence (2) is commutative. This completes the proof.

§6. Proposition 6.1. If $B / T$ is a trivial finite $G$-Galois extension then $P_{K}\left(\Delta_{1} / B\right)^{(G)} \rightarrow \operatorname{Pic}_{K}(B)^{G} \rightarrow 1$ is exact and splits, where $\Delta_{1}$ is a crossed product of $B$ and $G$ with trivial factor set $(C f .[16 ;$ Cor. 2].) 
Proof. $B$ is the direct sum of $(G: 1)$ copies of $T$. Put $e_{\sigma}=$ $(0, \cdots, 0,1,0, \cdots, 0)$ (the $\sigma$-component is 1 ). Then $\sum_{\sigma} e_{\sigma}=1, e_{\sigma} e_{\tau}=\delta_{\sigma, \tau} e_{\sigma}$, and $B=\sum \oplus T e_{\sigma}$. The operation of $G$ on $B$ is given by $\tau\left(e_{\sigma}\right)=e_{\tau \sigma}$. Let $[P] \in \mathrm{Pic}_{K}(B)^{G}$. Then ${ }_{B} B u_{\sigma} \otimes{ }_{B} P_{B} \stackrel{\approx}{\longrightarrow} P \otimes{ }_{B} B u_{\sigma_{B}}$ for all $\sigma \in G$. Multiplying $e_{1}$ on the right, we have ${ }_{B} B u_{\sigma} e_{1} \otimes{ }_{B} e_{1} P_{B} \stackrel{\approx}{\longrightarrow}{ }_{B} P e_{\sigma} \otimes{ }_{B} e_{\sigma} B u_{\sigma_{B}}$ for all $\sigma \in G$. Hence $h_{\sigma}:{ }_{T} e_{1} P_{T} \stackrel{\approx}{\longrightarrow}{ }_{T} e_{\sigma} P_{T}$ for all $\sigma \in G$, because ${ }_{T} e_{\sigma} B_{T}=$ ${ }_{T} e_{\sigma} T_{T} \stackrel{\approx}{\longrightarrow} T_{T}, e_{\sigma} t \mapsto t(t \in T)$. It is easily seen that $\left[e_{1} P\right] \in \operatorname{Pic}_{F}(T)$, where $F$ is the center of $T$. Put $e_{1} P=P_{0}$, and let $\left(P_{0}\right)_{G}$ be the module of all $G \times G$ matrices over $P_{0}$, and let $P^{\prime}$ be its diagonal part. Then it is evident that $\left(P_{0}\right)_{G}$ is canonically a two-sided $(T)_{G}$-Morita module, where $(T)_{G}$ is the ring of all $G \times G$ matrices over $T$. Indifying $B$ with the diagonal part of $(T)_{G},{ }_{B} P_{B}^{\prime}$ is isomorphic to ${ }_{B} P_{B}$. And $(T)_{G} \otimes{ }_{B} P^{\prime} \stackrel{\approx}{\longrightarrow}\left(P_{0}\right)_{G}$ as left $(T)_{G}$, right $B$-modules, canonically. Since $e_{\sigma}(\sigma \in G)$ is a basis for $B_{T}, \Delta_{1}=\operatorname{Hom}_{l}\left(B_{T}, B_{T}\right) \stackrel{\approx}{\longrightarrow}(T)_{G}$. Then we can easily see that the canonical $\operatorname{map} P^{\prime} \rightarrow(T)_{G} \otimes_{B} P^{\prime}$ is in $P_{K}\left((T)_{G} / B\right)^{(G)}$.

Proposition 6.2. If $\Delta / B$ is a group ring then the sequence $P_{K}(\Delta / B)$ $\rightarrow \operatorname{Pic}_{K}(B) \rightarrow 1$ is exact, and splits.

Proof. Let $[P] \in \mathrm{Pic}_{K}(B)$. Then there is a $B$-B-isomorphism $B G \otimes{ }_{B} P \rightarrow P \otimes{ }_{B} B G, \sigma \otimes p \mapsto p \otimes \sigma(\sigma \in G)$, and this isomorphism satisfies the condition in Lemma 1.2.

Remark. The above proposition can be generalized to the case that $\Delta=\sum \oplus B u_{\sigma}, u_{\sigma} b=b u_{\sigma}(b \in B), u_{\sigma} u_{\tau}=a_{\sigma, \tau} u_{\sigma \tau}$ with $a_{\sigma, \tau} \in U(K)$. The proof is analogous to the above one.

Proposition 6.3. Let $A, B, L$, and $K$ be rings as in $\S 2$, and fix a group homomorphism $J: G \rightarrow \circlearrowleft(A / B)$. Suppose that $B / K$ is separable and that $K \subseteq L$. Then

$$
P_{K}(A / B)^{(G)} \stackrel{\approx}{\longrightarrow} \operatorname{Aut}(A / B)^{(G)} \times \operatorname{Pic}_{K}(K),
$$

and this induces

$$
P^{L}(A / B)^{(G)} \stackrel{\approx}{ } \operatorname{Aut}(A / B \cdot L)^{(G)} \times \operatorname{Pic}_{K}(K) .
$$

Proof. Let $\phi: P \rightarrow M$ be in $P_{K}(A / B)$. Then there is an automorphism $f$ of $V_{A}(B) / K$ such that $f(c) \phi(p)=\phi(p) c$ for any $c \in V_{A}(B), p \in P$, and the $\operatorname{map}[\phi] \mapsto f$ is a group homomorphism from $P_{K}(A / B)$ to $\operatorname{Aut}\left(V_{A}(B) / K\right)$ (cf. [19; Prop. 3.3]). Then the map Aut $(A / B) \rightarrow P_{K}(A / B) \rightarrow \operatorname{Aut}\left(V_{A}(B) / K\right)$ 
is the restriction to $V_{A}(B)$. Let $U$ be a $B$-B-module such that $b u=u b$ for all $b \in K, u \in U$. Put $B^{e}=B \otimes{ }_{K} B^{0 P}$. Then $U$ may be considered as a left $B^{e}$-module. By [14; Th. 1.1], ${ }_{B} U \stackrel{\approx}{\longrightarrow} \operatorname{Hom}_{r}\left({ }_{B} B^{e}{ }_{{ }_{B} e} B\right) \otimes{ }_{K} \operatorname{Hom}_{r}\left({ }_{B} B{ }_{B_{B} e} U\right)$, and so $U=B \otimes_{K} V_{U}(B)$. In particular, $A=B \otimes_{K} V_{A}(B)$. Hence $\operatorname{Aut}(A / B)$ $\stackrel{\sim}{\longrightarrow} \operatorname{Aut}\left(V_{A}(B) / K\right)$ by restriction. Let $\bar{f} \mid V_{A}(B)=f$, and assume that $\phi \in P_{K}(A / B)^{(G)}$. Then $J_{\sigma} \cdot \phi(P)=\phi(P) J_{\sigma}=\bar{f}\left(J_{\sigma}\right) \phi(P)$, because $J_{\sigma}=$ $B \cdot V_{J_{\sigma}}(B)$. Hence $\bar{f}\left(J_{\sigma}\right)=J_{\sigma}$ for all $\sigma \in G$. Therefore the image of $\phi$ in $\operatorname{Aut}(A / B)$ belongs to $\operatorname{Aut}(A / B)^{(G)}$. Hence the map Aut $(A / B)^{(G)}$ $\rightarrow P_{K}(A / B)^{(G)} \rightarrow$ Aut $(A / B)^{(G)}$ is the identity map. Combining this with Prop. 2.2, we know that $P_{K}(A / B)^{(G)} \stackrel{\approx}{\longrightarrow}$ Aut $(A / B)^{(G)} \times \operatorname{Im} \alpha$, where $\alpha: P_{K}(A / B)^{(G)} \rightarrow \operatorname{Pic}_{K}(B)^{G}$ is the one as in Prop. 2.2. By Remark to Lemma 2.4, $\operatorname{Pic}_{K}(K) \stackrel{\approx}{\longrightarrow} \operatorname{Pic}_{K}(B),\left[P_{0}\right] \mapsto\left[B \otimes{ }_{K} P_{0}\right]$. Then the canonical map $B \otimes$ ${ }_{K} P_{0} \rightarrow A \otimes{ }_{K} P_{0}$ is in $P_{K}(A / B)^{(G)}$. Therefore $\operatorname{Im} \alpha \stackrel{\approx}{\longrightarrow} \operatorname{Pic}_{K}(K)$. Thus we have the first assertion. The second assertion is obvious.

COROLlaRY. Let $L \supseteq K$ be commutative rings, and we fix a group homomorphism $G \rightarrow \operatorname{Aut}(L / K)$. Then

$$
P^{L}(L / K)^{(G)}=P^{L}(L / K) \stackrel{\approx}{\longrightarrow} \operatorname{Pic}_{K}(K) \text {. (cf. §3) }
$$

Proof. Let $\sigma \in G$. Then, for any $\left[P_{0}\right] \in \operatorname{Pic}_{K}(K),\left(L u_{\sigma} \otimes{ }_{K} P_{0}\right) \otimes{ }_{L} L u_{\sigma-1}$ $\stackrel{\approx}{\longrightarrow} L \otimes{ }_{K} P_{0}, x u_{\sigma} \otimes p_{0} \otimes u_{\sigma-1} y \mapsto x y \otimes p_{0}$, as $L$ - $L$-modules.

Remark. By the above Cor, the sequence

$$
\circlearrowleft(L / K)^{(G)} \longrightarrow P^{L}(L / K)^{(G)} \longrightarrow \operatorname{Pic}_{L}(L)^{G}
$$

is isomorphic to

$$
\text { ङS }(L / K)^{(G)} \longrightarrow \operatorname{Pic}_{K}(K) \longrightarrow \operatorname{Pic}_{L}(L)^{G} .
$$

(Cf. Th. 3.4, [8], and [16].)

Proposition 6.4. Let $A \supseteq B$ be rings, and $L$ the center of $A$. Assume that $A \otimes{ }_{L} V_{A}(B) \mid A$ as left $A$, right $V_{A}(B)$-modules, and $V_{A}\left(V_{A}(B)\right)=B$. Then

$$
P^{L}(A / B) \stackrel{\approx}{\longrightarrow}(A / B) \times \operatorname{Im} \alpha
$$

where $\alpha: P^{L}(A / B) \rightarrow \operatorname{Pic}_{L}(A)$ is the one as in Th. 3.4. (Cf. [14], [19].)

Proof. By [19; Th. 1.4], Aut $\left(V_{A}(B) / L\right) \stackrel{\approx}{\longrightarrow}(A / B)$, and the map 


$$
\mathbb{B S}(A / B) \longrightarrow P^{L}(A / B) \longrightarrow \operatorname{Aut}\left(V_{A}(B) / L\right) \stackrel{\approx}{\longrightarrow}(A / B)
$$

is the identity (cf. [19; Prop. 3.3]). Then, by Th. 1.4, we can complete the proof.

\section{REFERENCES}

[1] M. Auslander and O. Goldman: The Brauer group of a commutative ring, Trans. Amer. Math. Soc., 19 (1960), 367-409.

[2] G. Azumaya: Algebraic theory of simple rings (in Japanese), Kawade Syobô, Tokyo, 1952.

[ 3 ] G. Azumaya: Maximally central algebras, Nagoya Math. J., 2 (1951), 119-150.

[4] G. Azumaya: Completely faithful modules and self injective rings, Nagoya Math. J., 27 (1966), 697-708.

[ 5 ] H. Bass: The Morita Theorems, Lecture note at Univ. of Oregon, 1962.

[6] H. Bass: Lectures on topics in algebraic K-theory, Tata Institute of Fundamental Research, Bombay, 1967.

[7] H. Bass: Algebraic K-theory, Benjamin, 1968.

[8] S. U. Chase, D. K. Harrison and A. Rosenberg: Galois Theory and Galois cohomology of commutative rings, Mem. Amer. Math. Soc., 52 (1965).

[9] S. U. Chase and A. Rosenberg: Amitzur complex and Brauer group, Mem. Amer. Math. Soc., 52 (1965).

[10] F. R. DeMeyer: Some note on the general Galois theory of rings, Osaka J. Math., 2 (1965), 117-127.

[11] F. R. DeMeyer and E. Ingraham: Separable algebras over commutative rings, Springer, 1971.

[12] D. K. Harrison: Abelian extensions of commutative rings, Mem. Amer. Math. Soc., 52 (1965).

[13] K. Hirata: Some types of separable extensions of rings, Nagoya Math. J., 33 (1968), 108-115.

[14] K. Hirata: Separable extensions and centralizers of rings, Nagoya Math. J., 35 (1969), 31-45.

[15] T. Kanzaki: On Galois algebras over a commutative ring, Osaka J. Math., 2 (1965), 309-317.

[16] T. Kanzaki: On generalized crossed product and Brauer group, Osaka J. Math., 5 (1968), 175-188.

[17] Y. Miyashita: Finite outer Galois theory of non-commutative rings, J. Fac. Sci. Hokkaido Univ., Ser. I, 19 (1966), 114-134.

[18] Y. Miyashita: Galois extensions and crossed products, J. Fac. Sci. Hokkaido Univ., Ser. I, 20 (1968), 122-134.

[19] Y. Miyashita: On Galois extensions and crossed products, J. Fac. Sci. Hokkaido Univ., Ser. I, 21 (1970), 97-121.

[20] K. Morita: Duality for modules and its application to the theory of rings with minimum condition, Sci. Rep. Tokyo Kyôiku Daigaku, 6 (1958), 83-142. 